UCRL-CR-121458

S/C-B239634

\title{
Theoretical Description of Laser Melt Pool Dynamics Task Order Number B239634 \\ Quarter 3 Report
}

\section{A. Dykhne}

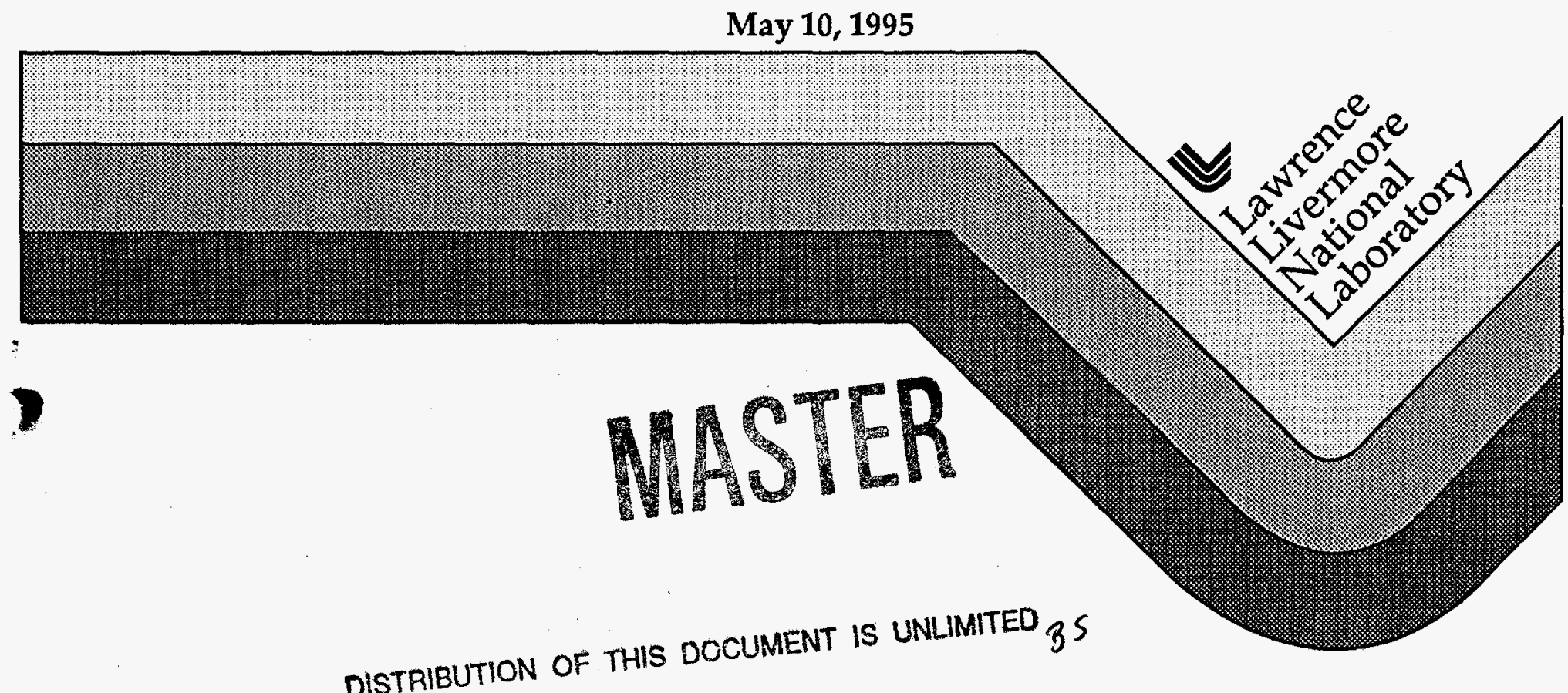




\section{DISCLAIMER}

Work performed under the auspices of the U.S. Department of Energy by Lawrence Livermore National Laboratory under contract number W-7405-ENG-48.

This document was prepared as an account of work sponsored by an agency of the United States Government. Neither the United States Government nor the University of California nor any of their employees, makes any warranty, express or implied, or assumes any legal liability or responsibility for the accuracy, completeness, or usefulness of any information, apparatus, product, or process disclosed, or represents that its use would not infringe privately owned rights. Reference herein to any specific commercial products, process, or service by trade name, trademark, manufacturer, or otherwise, does not necessarily constitute or imply its endorsement, recommendation, or favoring by the United States Government or the University of California. The views and opinions of authors expressed herein do not necessarily state or reflect those of the United States Government or the University of California, and shall not be used for advertising or product endorsement purposes. 


\section{DISCLAIMER}

Portions of this document may be illegible in electronic image products. Images are produced from the best available original document. 


\section{State Research Center of the Russian Federation Troitsk Institute for Innovation and Thermonuclear Investigation ${ }^{3}$}

\section{THEORETICAL DESCRIPTION OF LASER MELT POOL DYNAMICS \\ TASK ORDER NUMBER B239634 QUARTER 3 REPORT}

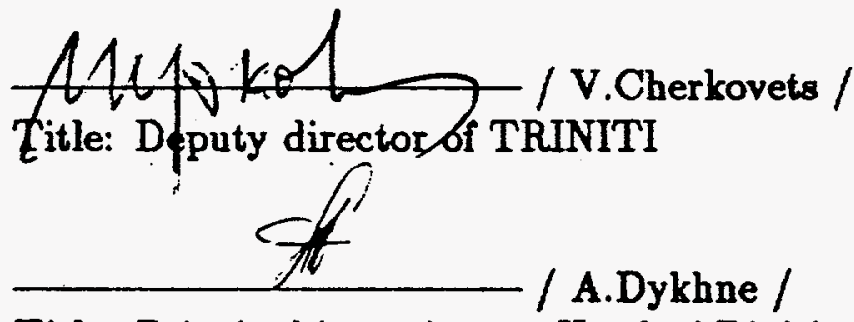

Title: Principal investigator, Head of Division of TRINITI Date: .1............... 1895

\footnotetext{
${ }^{1}$ Troitsk, Moscow region, Rusgia Fax:(095)334-51-58,e-mail:likhanskOanet.80vam com
} 
List of investigators

V. Likhanskii............. Head of Department

A. Loboiko.................. Senior Scientist

V. Kiselev.................. Senior Scientist

V. Vityukov................ Scientist

G. Gladush............... Senior Scientist

E. Loboiko................. Engineer 


\section{Contents}

1 Introduction 4

2 Regimes of hydrodynamic removal of melted material 5

2.1 Closed hydrodynamic flows .............. 5

2.2 Closed flows at melting with evaporation ........ 7

2.3 Unclosed flows ................. 8

3 Melt motion 9

3.1 Theoretical consideration and classification of melt removal

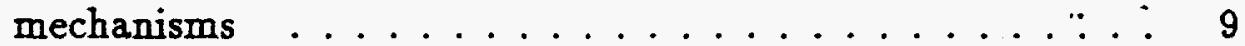

4 Marangony effect $\quad 10$

4.1 Theoretical consideration of the Marangony effect ..... 10

4.2 Calculation of mass transfer in case of laser doping ..... . 13

5 Material ablation under conditions of intense laser-induced evaporation

5.1 Theoretical consideration of the ablation process . . . . . 15

5.2 Processes in gaseous ambience ............ 18

5.3 Numerical modelling of the laser ablation process . . . . . 21

6 Conclusion 23 


\section{Introduction}

Melting of solid matter under laser radiation is realized almost in every process of laser technology $[1,2,3]$. First of all, this is welding, drilling, cutting, alloying. Obtaining of big depths of quenching is usually followed by melting of sample surface layer. Therefore, study of behavior of liquid in laser melt is important for understanding of physical processes and mechanisms of laser technology. Hydrodynamics of liquid has, of course, specific features at different technological processes but it may have a number of common features. From the viewpoint of liquid flow structure in laser melt one may outline two types of flows: with closed flow lines and unclosed flow lines and also more complicated flows when in a liquid bath both types of flow exist. Closed vortex flows may be developed at quenching and alloying of metals using pulsed laser beam. At the same technological processes using continuous wave or repetitive pulse laser beam the flow in the melt bath may already be more complicated. The same complicated flow with closed and unclosed flow lines is realized at laser welding. The flow with unclosed lines takes place in processes connected with material removal from the sample under processing, for example, while metal cutting and drilling.

At high flux densities, the greater part of energy may be spent for the evaporation process and material removal from the surface being heated. The role of hydrodynamic heat and mass transfer decreases due to the following two reasons. An increase in the laser energy absorbed by the surface causes, on the one hand, intensification of the vapor flow and a rise in energy and mass transfer due to the latter and, on the other hand, an enhancement of the vapor pressure and a drop in the melted layer thickness. In addition, the function of viscosity that reduces flow velocities enhances as well.

The present paper addresses melted material flows in cases when melt zones are shallow, i.e., the zone width is appreciably greater than or of the same order as its depth. Such conditions are usually realized when hardening, doping or perforating thin plates or when using none-deep penetration. Melted material flowing under conditions of deep penetration, drilling of deep openings and cutting depends on a number of additional factors (as compared to the shallow-pool case), namely, formation of a vapor and gas cavern in the sample and propagation of the laser beam through the cavern. These extra circumstances complicate hydrodynamic consideration of the liquid bath and 
will be addressed in the paper to follow.

\section{Regimes of hydrodynamic removal of melted material}

\subsection{Closed hydrodynamic flows}

Liquid vortex flows due to their closeness on the average do not result in target mass transfer but they influence on heat and impurities transfer inwards the target and, as a result, may define such important parameters of technological processes like quenching depth and alloying depth. On the face of it, one may suppose that the influence of such flows on this processes is reduced to the increase of thermal conductivity as well as diffusion coefficient of melted material. In this case, at least in steady-state problems the temperature profile is the only one to be changed in liquid bath. Temperature distribution in solid material $\left(\mathrm{T}<\mathrm{T}_{\text {melt }}\right)$ and liquid bath shape are not changed [2]. However, in the picture of paraffin melt irradiated by low-power continuous-wave $\mathrm{CO}_{2}$-laser $(20 \mathrm{~W})$, it is distinctly seen that vortex flow significantly changes the melt bath shape [2]. This shape qualitatively differs from the one that should have been set in under the influence of usual thermal conductivity, i.e. half-sphere ( for the case when the melt zone size are considerably bigger than laser beam radius ).

This simple and obvious experiment shows how important the role may be of vortex flows in formation of melt bath. If one needs to substantiate the basic role of vortex flows in heat transfer inside the sample, then, the crucial impact of vortex on alloyed impurity transfer from sample surface inside the melt is more or less evident. The estimates of penetration depths of alloyed impurity in terms of molecular diffusion give values lower than that obtained in experiment by an order of magnitude. However, one should remember that in laminar flow the transfer of impurities and their distribution over bath depend on joint impact of convective transfer and molecular diffusion. And, generally speaking, it is not evident that origination of closed flows in the melt bath will always provide alloyed impurities transfer from the

surface to the very bottom of bath. From the mentioned above it follows that to create a model of alloying and quenching (with melting) the numerical 
simulations are necessary together with analytical study, since one has to consider, in particular, 2-D processes of heat-mass transfer at the background of complicated hydrodynamic flows.

Presently, three mechanisms are discussed in literature of closed flows excitation in laser melt:

1. on account of Marangony effect

2. on account of non steady-state melting

3. on account of gas flow excitation along the melt surface.

But all these mechanisms begin to operate after melting has begun. Therefore, we begin theoretical consideration of origination of flows from clearing up the conditions of material melting. Assessment formulas will be helpful for analysis of numerical computing results also.

So, if laser beam having intensity $q$ ( further on $q$ implies the absorbed intensity ) was incident on flat surface, then by moment $t$ the heat wave propagates at depth $2 \sqrt{\chi^{t}}$. If $2 \sqrt{\chi^{t}} \ll r_{b}$ the heat problem may be considered to be 1-D. The surface temperature $T$, is defined from heat balance condition at the boundary

$$
q=k \frac{T}{2 \sqrt{\chi^{t}}}
$$

From this, we obtain a known result that in the case of flat surface the surface temperature grows versus time $\sim \sqrt{t}$ :

$$
T_{s}=\frac{q}{c \rho} \sqrt{4 t / \chi}
$$

where $\mathbf{k}$ is thermal conductivity, $\chi$ is temperature conductivity, $\rho$ is the density and $c$ is specific heat capacity, $\mathrm{r}_{b}$-laser beam radius.

Temperature reduction inwards material is known to be described by error function which may be replaced by exponent $T \leq T$, to facilitate approximate calculations:

$$
T(z)=\frac{q}{c \rho} \sqrt{4 t / \chi} \exp (-z / \sqrt{4 t \chi})
$$

Using (3) one can readily write an expression for melt depth

$$
H_{\text {melt }}=2 \sqrt{\chi t} \ln \left(\frac{q}{c \rho T_{\text {melt }}} \sqrt{4 t / \chi}\right)
$$


where $T_{\text {melt }}$ is the temperature of melting.

From (2), (4) one may define the melt depth by the moment of the beginning of boiling

$$
\left(H_{\text {melt }}\right)_{\max }=\frac{c \rho \chi T_{\text {boil }}}{q} \ln \left(\frac{T_{\text {boil }}}{T_{\text {melt }}}\right)
$$

where $T_{\text {boil }}$ is the temperature of boiling.

It is seen using (5) that to achieve the maximum melt depth by the moment of liquid boiling one should escape high intensities of laser beam. Another limitation for expression (4) may be setting in the steady-state temperature distribution when $t>r_{b}^{2} / \chi$. 1-D cases are usually realized at laser alloying.

\subsection{Closed flows at melting with evaporation}

As it is seen from (5) at high values of intensity, boiling up occurs already at small melting depths. The pressure of recoil vapors may significantly exceed the thermo-capillary force. But as it is known, the force of pressure is normal to liquid surface and on the face of it must not cause shear flows of liquid. However, more detailed consideration of melting demonstrates that melt flow that gathers speed due to pressure of vapor upon free surface also has vortex component [3]. This is connected with the fact that speeding up of the melt and melting itself that increases the depth of liquid, take place simultaneously, provided that metal layers melted at later moments of time absorb smaller pulse than that located closer to the surface and are, of course, speeded up to lower velocities. In present speculations, the liquid is supposed not to splash out of the bath and upper and lower layers counteracts each other. According to the noted above the upper layer having obtained greater force momentum moves away from the center and makes the lower layer flowing in the opposite direction i.e. vortex movement arises. The condition when surface tension prevents splash out is as follows:

$$
\frac{2 \sigma}{\Delta \ell}>P
$$

If to evaluate the vapor pressure $P$ for the case when all laser radiation is spent on evaporation $\left(P=q C_{s} / L_{s}\right)$ and to take as $\Delta \ell$ the melt depth by 
the moment of boiling up (5), then, the condition of absence of splash out turns out to be independent of intensity

$$
\frac{2 L_{s} \sigma}{k C, T_{\text {boil }} \ln \left(T_{\text {boil }} / T_{\text {melt }}\right)} \geq 1
$$

where $C_{\mathrm{z}}$ is sonic velocity, $L_{\mathrm{a}}$ - specific heat of sublimation.

For example, this condition is not valid for iron since the left part equals to $1 / 3$. Thus, if the discussed mechanism of vortex excitation takes place, it is either together with splash out or at moments of time preceding heavy boiling, when vapor pressure is not quite high. Therefore, to answer these questions numerical tests are necessary.

\subsection{Unclosed flows}

Unclosed flows lead either to material removal from the target or to displacement of it beyond the limits of heated zone of interaction where it hardens. Excitation of such flows is mainly influenced by the pressure of material vapor. In the first part of report, the laws of vapor origination and their impact on the target were discussed in details. As an example of such impact, a theoretical model of pulsed removal of melt under pressure of vapor recoil was considered. This is a typical example of unclosed flow when the liquid is splashed out of the interaction zone.

Several cases of material removal from liquid bath on account of pressure of vapor recoil may be realized, differing from each other by internal mechanism of melt removal: established mode, non established mode, mode of splash out when the radiation is switched off and the liquid moves under its own momentum. Besides, the liquid film may be removed remaining homogeneous in thickness or inhomogeneous. All these cases differ from one another by a specific damage energy, respectively, the value that is of practical importance since it defines the efficiency of technological process. Naturally, the mechanism of removal affects upon the quality of treatment. 


\section{$3 \quad$ Melt motion}

\subsection{Theoretical consideration and classification of melt removal mechanisms}

We believe that it is convenient and evident to present this qualification in Fig. 1 in coordinates $\epsilon, \tau$; where $\epsilon$ - absorbed energy density of laser beam during pulse, $\tau-$ pulse duration. Application scheme is presented for a definite target material and for a given size of light spot on the target. The necessity of 2-D representation is stipulated by the fact that replacing of one mechanism by another depends on a number of parameters. The values $\epsilon$ and $\tau$ are the main variables. The analysis is of qualitative character and does not describe transitional stages from one mode to another. "To excite liquid movement it is necessary that at least by the end of pulse the liquid boiling begins. To meet this requirement, the pulse energy $\epsilon$ must exceed the energy of material layer heating under the spot up to boiling temperature

$$
\epsilon>\epsilon_{c r}=c \rho T_{b o i l} \sqrt{\chi \tau}
$$

In order the non steady-state mode (splash mode) described in the first part does not turn into steady-state wave mode at increase of $\epsilon$, it is necessary [2] that

$$
\frac{\epsilon}{\tau}<q_{2}=\left[c \rho T_{b o i l} \sqrt{2 \chi / r_{b}}\right]^{4 / 3}\left[\frac{2 c_{s}}{\rho L_{s}}\right]^{1 / 3}
$$

If $\epsilon / \tau>q_{2}$ then the gushing wave is realized which at $\epsilon / \tau>q_{1}$ turns into mass removal mode on account of evaporation (evaporation wave [6])

$$
q_{1}=\rho L,\left[c_{s} \chi^{2} / r_{b}^{2}\right]^{1 / 3}
$$

The value $q_{1}>q_{2}$, since $q_{2} / q_{2}=\left[L_{0} / 2 c T_{\text {boil }}\right]>1$.

Two more curves should be put in Fig.1. The curve $\epsilon^{\prime}$ designates that by the end of pulse the liquid from the bath is totally removed due to splash mode [2]. If $\epsilon^{\prime}>\epsilon$, then, by the end of pulse the liquid has no time to leave the bath and is removed under its own momentum. If $\epsilon>\epsilon^{\prime}$, then, liquid removal due to splash mode takes place before pulse termination and until pulse termination the liquid is removed due to gushing wave. 
Finally, curve $\sqrt{2} \epsilon_{\text {er }}$ shows that at $\sqrt{2} \epsilon_{c r}<\epsilon$, surface boiling up takes place inhomogeneously ( first, in the center) and at $\epsilon>\sqrt{2} \epsilon_{c r}$ the entire zone of interaction begins boiling almost simultaneously. Therefore, a nonhomogeneous splash under one's own momentum must be realized in zone $\mathrm{V}$, and nonhomogeneous removal due to splash and gushing wave in zone IV. Zone III - removal on account of homogeneous splash and gushing wave. Expressions for critical $\tau_{1}, \tau_{2}$ are as follows

$$
\tau_{1}=\left[\frac{c T_{\text {boil }}}{L_{s}}\right]^{2}\left[\frac{r_{b}^{2}}{\chi c_{a}^{2}}\right]^{1 / 3}, \tau_{2}=\left[\frac{L_{s}}{c T_{\text {boil }}}\right]^{2}\left[\frac{r_{b}^{2}}{\chi c_{a}^{2}}\right]^{1 / 3}
$$

i.e. $\tau_{1}<\tau_{2}$. For example, for steel $\left(r_{b}=0,1 \mathrm{~cm}\right) \tau_{1}=5 \mathrm{mks}, \tau_{2}=100 \mathrm{mks}$.

Thus, from this scheme it follows that at $\tau<\tau_{1}$ material removal is realized only due to evaporation, independently of pulse energy. To realize most effective modes of splash, it is necessary (but not enough) to work at $\tau>\tau_{2}$. It is evident that the scheme is qualitative and helps to determine nothing more but tendencies at interaction parameters variation.

\section{Marangony effect}

\subsection{Theoretical consideration of the Marangony ef- fect}

This effect is stipulated by origination of shift thermo-capillar forces arising due to dependence of surface tension on temperature [4]. In the case of metals, tension decreases versus temperature. In conditions of laser technology, heated zone size is not big (as it was mentioned above they are close to focus spot size) temperature gradients are big and one may expect rather vivid manifestation of this effect. In conditions of laser technology, material melting and the impact of this effect occur simultaneously that complicates the consideration of arising flows. However, in metals, for example, temperature deffusivity $\chi$ is bigger than kinematic viscosity $\nu$ (Prandtl number, $P r=\nu(\chi \sim 0,1)$. Due to it the thermal wave propagates faster than viscous wave, therefore, to proper analyze it is possible to separate these problems.

Like thermal problem the problem of thermo-cappillar convection has two stages: non steady-state and steady-state. They may be studied by analytical 
methods only separately from one another. For the narration to be brief and clear enough we shall limit ourselves to a qualitative description of thermocapillar convection mechanism. So, the force affecting a unit surface of liquid bath, $\partial \sigma / \partial x$, is balanced by viscous friction force $\eta \partial V_{x} / \partial z$

$$
\eta \partial V_{x} / \partial z=\partial \sigma / \partial x \equiv(\partial \sigma / \partial T)(\partial T / \partial x)
$$

where $\sigma$ is surface tension coefficient, $\eta$ is dynamic viscosity.

Thus, rotor of velocity is given on liquid surface. Until velocity values are small and, hence, Reinolds number $(\operatorname{Re} \ll 1)$ is small, the variation of velocity rotor is expressed by Helmholtz equation.

$$
\frac{\partial}{\partial t}(\operatorname{rot} V)=\nu \Delta(\operatorname{rot} V)
$$

This equation is similar to heat propagation equation. As we know, in non steady-state flat case, the heat during time $t$ propagates through depth $2 \sqrt{\chi t}$. The same way, the area of surface force impact may be written as $\delta_{\eta}=2 \sqrt{\nu t}$. Knowing $\delta_{\eta}$ one may derive the surface velocity from (6)

$$
V_{s}=\frac{1}{\eta}(\partial \sigma / \partial T)(\partial T / \partial x) \sqrt{\nu t}
$$

From a continuity condition of mass flow $V_{s} \delta=V_{x} H_{\text {melt }}$ we may find the velocity inside the molten pool

$$
V_{x}=\frac{t}{\rho H_{\text {melt }}}(\partial \sigma / \partial T)(\partial T / \partial x)
$$

When boundary layers are closed, i.e. at $t=H_{\text {melt }}^{2} / \nu$, the known steadystate viscous thermo-capillary flow is established in the system [4]

$$
V_{x}=\frac{H_{m e l t}}{\eta}(\partial \sigma / \partial T)(\partial T / \partial x)
$$

However, in conditions of laser technology (due to big values of $\partial T / \partial x$ ) before this steady-state mode is achieved the nonlinear terms of Navie-Stoke's equation begin to operate since Reynolds and Peclet numbers become big.

One may suppose that in this case the general flow structure is not changed (3) but the ratios will be different. In course of fast rotation, a liquid particle undergoes alternating heat influence. Close to the bath surface it is 
heated but when it moves along walls it is cooled. Per a single rotation a perturbation penetrates inside the liquid at a specific depth $\delta_{T} \simeq \sqrt{\chi / \omega}$, $\omega$-rotation frequency. Therefore, the more the rotation velocity is the thinner the heat boundary layer is $i . e$. the characteristic size of temperature decrease $\Delta T$ is $\delta_{T}$. At a given intensity $q$

$$
\Delta T=q \delta_{T} / k
$$

I.e. the temperature decrease compared to a motionless (or slowly rotating) liquid is reduced $\sqrt{\operatorname{RePr}}$ times. In particular case of liquid rotation similar to solid-state matter rotation, this conclusion is proved strictly [5].

The same picture must occur in velocity distribution as well. Rotor of velocity in the volume of bath is constant and varies only within a narrow area of viscous boundary layers [5]

$$
\delta_{\eta} \simeq d / \sqrt{R e}
$$

The same as (8) was derived, if to equalize surface tension to friction taking into account specific size of velocity and temperature variation, one can easily get:

$$
V=\left|\frac{\partial \sigma}{\partial T}\right| \frac{q}{\eta d} \delta_{T} \delta_{\eta} \frac{1}{k}
$$

Substituting expressions for $\delta_{T}$ and $\delta_{\eta}$ in (13) we get ultimately

$$
V^{2}=\left|\frac{\partial \sigma}{\partial T}\right| \frac{q}{k \rho \sqrt{\mathrm{Pr}_{\mathrm{r}}}}
$$

From (14) it is seen that due to temperature gradient reduction as a result of convection in the bath, liquid velocity depends on intensity not linearly but weaker: like $\sqrt{q}$.

Thus, the phenomena of excitation as well as steady-state picture of closed vortex flows are studied analytically at least in fragments. Of course, a number of parameters included in formulas, for example, bath depth, especially at $H \simeq d$ remains indefinite and the final word rests with numerical results. But analytical expression are very useful for both: clear understanding of processes and comprehension of numerical results as well as for assessments of experiments planned. 


\subsection{Calculation of mass transfer in case of laser dop- ing}

Numerical calculation of thermocapillary convection for the case of laserassisted metal doping are of significance from the viewpoint of both better understanding of physical aspects of the phenomenon and prediction of experimental results. However, comparison of such calculations with the experiments can be more often than not only qualitative. Such a situation is chiefly due to the lack of relevant experimental data required for modelling the experiment under consideration. In particular, when performing laserassisted doping of metals, the radiation absorption coefficient is nearly never. measured in spite of the fact that it determines the temperature distribution throughout the sample surface. In its turn, the velocity distribution over the melt surface depends on the temperature gradient. As is known, dopant transfer deep into the melt bath is predominantly due to evolving convective flows of melted material. Therefore, the temperature dependence of the absorption coefficient is a very significant characteristic necessary for performing quantitative comparison of numerical calculation results with experimental data. Otherwise, only qualitative comparison can be accomplished.

We have modelled the experiment [13] on doping iron with molybdenum from a pre-deposited coating $30 \mu m$ thick. The two-layer system obtained was processed by a neodymium laser generating free-running mode single pulses with a wavelength of $1.06 \mu \mathrm{m}$, an energy $E \sim 5 \div 15 \mathrm{~J}$, a duration of $4 \mathrm{~ms}$, and a focal spot radius $r_{o}=1.5 \mathrm{~mm}$. In Figs.2a,b, the photographs of the doped zone at $E=7 \mathrm{~J}$ and $10 \mathrm{~J}$ respectively are presented.

The modelling was carried out using the code described in the previous report, with an additional equation for evolution of the dopant concentration $C(t)$ :

$$
\frac{\partial C}{\partial t}+\operatorname{div}(C u)=\operatorname{div} D g r a d C
$$

where $u$ is the melt flow velocity, $D$ is the dopant diffusion coefficient (in the calculations, $D$ is invariant and equal to $\left.10^{-5} \mathrm{~cm}^{2} / \mathrm{s}\right)$.

The initial distribution $C(0)$ was in agreement with the experiment: 


$$
C=\left\{\begin{array}{llrr}
1, & \text { at } & 0 \leq y \leq 30 & \mu m \\
0, & \text { at } & y>30 & \mu m
\end{array}\right.
$$

Various thermal constants of the iron-molybdenum mixture were calculated as

$$
A_{F e-M o}=A_{M o} C+A_{F e}(1-C) .
$$

In Figs.3-7, the calculated melt bath shape, isolines of melt stream function, and molybdenum concentration levels are presented for different moments at a laser radiation energy $E=10 \mathrm{~J}$. As time elapses, the central melt zone broke free of the pre-deposited molybdenum coating. As this takes place, the molybdenum concentration in near-to-surface layers of the remelt zone became lower than that in the doped zone in the interior of the bath. In addition, the molybdenum concentration varied only slightly with time at the vortex center and rather significantly at the periphery. The comparison of the numerical results (Fig.7) with those of X-ray diffraction microanalysis of the area affected [13] (Fig.2) evidences that the molybdenum concentrations are in agreement. It is worth noting that a more laminated remelt structure obtained in the experiment is readily explicable by the fact that the calculation used a constant absorption coefficient whereas in actual conditions it usually increases with temperature. Hence, a greater temperature gradient on the sample surface was most likely realized in the experiment, which gave rise to more intense convective flows and more noticeable penetration of the area with a reduced molybdenum concentration into that with an increased one.

The photographs of transverse microsections offer an opportunity to obtain, in addition to some data on the relative Mo content in the melt zone, the maximum depth of dopant penetration. Assuming that the thickness of the pre-deposited molibdenum coating equals $30 \mu \mathrm{m}$, we can infer that the doping depth in the experiment is $h \sim 90 \div 100 \mu m$ (at a laser radiation energy $E=10 \mathrm{~J}$, see Fig.2) and the calculated value is $h \sim 90 \mu m$ (see Fig. 7 ). Thus, a satisfactory agreement between the numerical results and the experimental data is achieved. 


\section{Material ablation under conditions of in- tense laser-induced evaporation}

\subsection{Theoretical consideration of the ablation process}

The regularities of laser-induced ablation of condensed materials can be explained adequately on the basis of the surface evaporation model [6]. Under this model, evaporation proceeds from a thin surface layer of the condensed medium. The layer thickness is assumed to be equal to the interatomic spacing. Energy transfer from the volume absorbing the laser radiation to the surface layer is due to heat conductivity. As a rule, in metals, the skin layer thickness where heat is released is well below the characteristic size of laser pulse energy penetration. At a sufficiently high level of surface heat release, the melted layer thickness is insignificant and hydrodynamic flows in the melted film are negligible during the pulse. Within the afore-presented approximation, the equations describing the ablation front motion acquire the following form:

$$
\begin{gathered}
c \rho \frac{\partial T}{\partial t}=\kappa \Delta T+Q \\
Q=A q_{0}(y, t) \cdot K \exp [-K(x-X(y, t))], x \geq 0
\end{gathered}
$$

Here $X(y, t)$ is the coordinate of the interface between the condensed phase and the gaseous one; $K$ is the absorption coefficient; $q_{0}(y, t)$ is the laser energy flux density; $A$ is the absorbing ability of the surface. Generally, optical and thermal properties of materials are temperature dependent. Eqs. (16) should be supplemented with boundary conditions. At $x \rightarrow \infty$, we assume that $T=T_{\infty}$ is given, $T_{\infty} \ll T_{\text {boil }}$. The other two conditions are provided for the moving boundary $x=X(y, t)$. One of them links the normal (with respect to the boundary) component of the temperature gradient in the condensed phase with the energy spent for surface evaporation at a small curvature of the moving boundary. This condition can approximated as follows [7]:

$$
\kappa \frac{\partial T}{\partial x}=\lambda \rho \dot{X}
$$


at $x=X(y, t)$. The second boundary condition establishes the dependence of evaporation front motion velocity on the temperature of the condensed phase surface and can be derived from the evaporation kinetics equation [6]. If the pressure at the sample surface is much higher than the ambient one, we may assume that we have to do with vacuum evaporation. The mass flux and evaporation front velocity are proportional to the saturated vapor pressure $P_{s}(T)$ at the surface temperature [6]. To determine $P_{s}(T)$, the Einstein model of condensed phases [8] is sometimes used, within which the Einstein frequency is an adjustment parameter. However, we can employ also the following dependence:

$$
P_{s}(T)=P_{0} \exp \left(-\frac{\lambda_{1}}{T}+\frac{\lambda_{1}}{T_{\imath}}\right) \equiv P_{0}^{*} \exp \left(-\frac{\lambda_{1}}{T}\right),
$$

which includes two adjustment parameters $P_{0}^{*}$ and $\lambda_{1}$. Here $\lambda_{1} \equiv \lambda A_{m} / N_{a}$ has a meaning of the atomic evaporation heat; $P_{0}$ and $T_{\text {are respectively the }}$ normal pressure and the boiling point under normal pressure.

With allowance made for the effect of the finite curvature of the evaporation surface upon the saturated vapor pressure above the surface, we use the following equation for the evaporation rate:

$$
\dot{X}=U \exp \left\{-\frac{1}{k T(x, y, t)}\left[\lambda_{1}+\frac{\sigma(T)}{n_{0}}\left(\frac{1}{R_{1}}+\frac{1}{R_{2}}\right)\right]\right\},
$$

where $U$ is a constant whose order of magnitude equals that of the sonic speed in the condensed medium; $R_{1}, R_{2}$ are the basic radii of surface curvature at the given point; $\sigma$ is the surface tension coefficient; $n_{o}$ is the number of atoms (molecules) in the unit volume of the condensed phase.

Under the afore-given approximation, the set of (16) - (19) determines the dynamics of the ablation wave front. Of interest seems to be an unidimensional quasistationary solution in the form of a plane wave propagating with a constant velocity $V_{o}$. Let us introduce the variable:

$$
\xi=x-V_{0} t .
$$

Then the quasistationary equation is as follows:

$$
-V_{0} c \rho \frac{\partial T}{\partial \xi}=\kappa \frac{\partial^{2} T}{\partial \xi^{2}}+A q_{0} K \exp (-K \xi)
$$


After integrating over $\xi$ from 0 to $+\infty$, we have:

$$
V_{0} \rho c\left[T(0)-T_{\infty}\right]=-\left.\kappa \frac{\partial T}{\partial \xi}\right|_{\xi=0}+A q_{0},
$$

and, using boundary condition (17), we get:

$$
\rho V_{0}\left[c\left[T(0)-T_{\infty}\right]+\lambda\right]=A q_{0}
$$

Let us consider the parameters in the expression above for the two metals - aluminum and iron. For aluminum, we have:

$$
T_{\text {melt }}=660.24^{\circ} \mathrm{C}, \quad T_{\text {boil }}=2520^{\circ} \mathrm{C}, \quad C_{p} \simeq(850 \div 1200) \frac{\mathrm{J}}{\mathrm{kgK} .} .
$$

(in the range of solid and liquid phases);

$$
\Delta H_{\text {melt }} \simeq 813 \frac{K J}{\mathrm{~kg}} ; \quad \Delta H_{\text {boil }} \simeq 2.2 \cdot 10^{4} \frac{\mathrm{KJ}}{\mathrm{kg}} .
$$

The parameters of iron are as follows:

$$
\begin{gathered}
T_{\text {melt }}=1538^{\circ} \mathrm{C} ; \quad T_{\text {boil }}=2872^{\circ} \mathrm{C} ; \\
C_{p}=(420 \div 1000) \frac{\mathrm{J}}{\mathrm{kgK}} ; \quad \Delta H_{\text {melt }}=525 \frac{\mathrm{KJ}}{\mathrm{kg}} ; \\
\Delta H_{\text {boil }}=1.33 \cdot 10^{4} \frac{\mathrm{KJ}}{\mathrm{kg}} .
\end{gathered}
$$

For metals (and not only for metals), rather representative are such relations between the parameters that the greater part of the energy spent for heating, melting and evaporation is expended for the evaporation process. Therefore, we can write approximate expressions for the ablation front velocity $V$ and the surface temperature:

$$
V_{0} \simeq \frac{A q_{0}}{\rho \lambda} \text { and } T(0)=\frac{\lambda_{1}}{K}\left[\ln \left(\frac{\rho \lambda U}{A q_{0}}\right)\right]^{-1}
$$

The value of heat flux through the boundary $\left.\frac{\partial T}{\partial \xi}\right|_{\xi=0}$ can be determined from (17), (22): 


$$
\left.\frac{\partial T}{\partial \xi}\right|_{\xi=0} \simeq \frac{1}{\kappa} \lambda \rho \frac{A q_{0}}{\lambda \rho} \simeq \frac{A q_{0}}{\kappa}
$$

If the saturated vapor pressure $P_{*}(T)$ at $T=T_{0}\left(T_{2}\right.$ is the surface temperature) is much greater than the ambient one, which is usually true for actual experiments, the reactive vapor pressure can be described (with an accuracy of several percent) by the vacuum evaporation dependence [6], i.e., $P=0.5 P_{\text {s. }}$. It is worth noting that the maximum of the temperature distribution is attained in the bulk of the sample:

$$
T(\xi)=-\frac{A q}{\kappa}\left(K-\frac{V_{0} \rho c}{\kappa}\right)^{-1} \exp (-K \xi)+\alpha \exp \left(-\frac{V_{0} \rho c}{\kappa} \xi\right)+T_{\infty}
$$

where

$$
\alpha=T(0)+\frac{A q}{\kappa}\left(K-\frac{V_{0} \rho c}{\kappa}\right)^{-1}
$$

Such a distribution is open to development of evaporation front instabilities.

\subsection{Processes in gaseous ambience}

The processes of radiation interaction with gases adjacent to the surface under irradiation can change both the structure of radiation that reaches the solid-phase boundary and the amount of energy absorbed. The field structure alteration is most significant under conditions favoring the development of near-to-surface optical breakdown plasma. The physical mechanisms governing the initial stage of gas breakdown are of a great variety and treated at length in literature (e.g., [9]).

Let us consider the dependence of the breakdown threshold versus the carrier frequency of radiation and the gas pressure. The electron-to-gas collision rate is $\nu=\langle\sigma v\rangle n$, where $n$ is the particles density, $\sigma$ is the collision cross-section, $v$ is the electron velocity, $<>$ means averaging over the velocities. Consequently, the collision rate is proportional to the pressure $P$. As an electron moves in the electromagentic wave field, it gains a certain mean energy 


$$
\epsilon_{a v}=(e E)^{2} / m\left(\omega^{2}+\nu^{2}\right) \cdot \frac{1}{\delta},
$$

where $m$ is the electron mass, $\sigma$ characterizes the inelastic loss due to collisions of the electron with atoms (molecules or ions). From the viewpoint of the elementary theory, the share of energy lost at a collision with a particle with mass $M$ equals $2 m / M$. If the gas pressure is very high $(\nu \gg \omega)$, the maximum achievable electron energy can be estimated from formula (24):

$$
\epsilon_{\max } \sim \frac{M E^{2}}{m \nu^{2}} \sim \frac{E^{2}}{P^{2}}
$$

If this energy value is under the ionization potential $\epsilon_{I}$ (from the ground or a sufficiently populated, excited state), the number of electrons cannot be increased. Therefore, at $\nu \gg \omega$ the threshold intensity of the laser-induced breakdown grows as $P^{2}$. It should be noted that the condition $\nu>\omega$ can be met only at high pressures (hundreds of atmospheres). So the case $\nu \ll \omega$ is a more interesting one for the problems of laser processing of materials.

Electrons from the area under irradiation can be lost due to diffusion $\nu_{d}=D / a^{2}$, where $D$ is the electron diffusion coefficient, $a$ is the characteristic size of the volume under irradiation. If, aside from diffusion, there are no other channels through which the electrons can be lost and these latter suffer energy loss through atom ionization, the electrons gain the energy sufficient for atom ionization in the time

$$
t_{u}=\epsilon_{I} \frac{m \omega^{2}}{e^{2} E^{2} \nu}
$$

To increase the number of electrons, $t_{u}$ must exceed the diffusion time

$$
\left(\nu_{d}\right)^{-1}=a^{2} / D
$$

From the condition above it follows that

$$
E_{t h}^{2}=\frac{D m \omega^{2} \epsilon_{I}}{\nu e^{2} a^{2}} \sim \frac{\omega^{2}}{p^{2} a^{2}}
$$

Hence, the breakdown threshold declines as the pressure increases or the radiation wavelength rises in the range $\nu \gg \omega$. 
In Fig.8, presented are the results of experimental research into the thresholds of nitrogen, argon and helium breakdown by means of ruby laser pulses with a duration of $50 \mathrm{ps}$ [10]. All the curves have a distinct minimum, the pressure value at the minimum being in approximate agreement with the condition $\nu \simeq \omega$.

In the range of low pressures, the breakdown threshold intensity declines as the radiation wavelength increases. The relevant experimental data on atmospheric-pressure air from a number of papers are summarized in a single graph [9], Fig.9. These points correlate quite well with the dependence $I_{t h} \sim$ $\lambda^{-2}$.

When the laser radiation interacts with materials, the gas-volume breakdown is preceded by either formation of a hot dense vapor cloud or intense emission of electrons from the metal heated. As a result, the function of the diffusion loss becomes insignificant and the threshold of near-to-surface breakdown reduces. The threshold versus pressure dependence becomes rather weak. The low-pressure regime of near-to-surface breakdown was considered for the first time in [11]. To provide the breakdown at a relatively high initial concentration of electrons, it is sufficient to meet the condition that the rate $\Delta \epsilon_{E}$ of electron energy gaining be above the rate of the loss due to elastic collisions in the whole range of electron energies up to $\epsilon_{I}$ :

$$
\Delta \epsilon_{E} \nu \geq \delta \nu \epsilon_{I}
$$

So, $I_{t h} \sim \omega^{2}$ and does not depend on pressure in the range $\omega^{2} \gg \nu^{2}$. The threshold intensities determined from (25) constitute several $\mathrm{MW} / \mathrm{cm}^{2}$ at a wavelength of $10.6 \mu \mathrm{m}$, which is approximately two orders of magnitude below than $I_{t h}$ in the gas volume.

Near-to-surface plasma is formed when laser radiation with a moderate intensity $\left(10^{6} \div 10^{10} \mathrm{~W} / \mathrm{cm}^{2}\right)$ interacts with a material. Such a range is typical of various regimes of laser processing techniques using continuous-wave, pulse or pulse-repetitive lasers. The plasma affects laser processing of materials through a number of ways, namely:

- chemical processes at the "condensed medium - ambient gas" interface. If the sample remains solid while the plasma exists, some oxide films or those of other compounds may appear (such films are formed with the aid of the plasma). These films can change optical properties of the surface and, hence, 
the regime of its heating. If the plasma is produced in contact with the melt, the melted layer may be doped with chemically active elements penetrating from the plasma;

- plasma formation can change noticeably the spatial and temporal distribution of heat flux inwards the sample. The laser radiation flux changes are due to absorption and refraction in the plasma. Furthermore, plasma formation can create an extra source of surface heating via heat exchange between the plasma and the sample;

- spatial and temporal distribution of pressure over the sample surface, which is determined by gas dynamics in the plasma.

\subsection{Numerical modelling of the laser ablation pro- cess}

In [12], the dependence of the recoil impulse of metal target vapor on pulsed laser radiation energy was studied experimentally in a range of intensities $10^{5}$ to $10^{7} \mathrm{~W} / \mathrm{cm}^{2}$. The experiments were performed using a neodymium laser $(\lambda=1.06 \mu \mathrm{m})$ with a pulse duration of $0.7 \mathrm{~ms}$. The laser pulse shape is presented in Fig.10. The target with a radius of $R=0.4 \mathrm{~cm}$ had an opening with a radius of $r=0.025 \mathrm{~cm}$ at the center for lightguide insertion. After the experimental results having been processed, the dependence of vapor recoil impulse $J$ on laser radiation energy $E$ (normalized to the irradiation spot area $S$ ) was obtained, which appears as

$$
J / S=a(E / S)^{b},
$$

where $a$ and $b$ are the empirical constants (specific for each metal). In particular, for aluminum under an ambient pressure $P_{\infty}=1 \mathrm{~Pa}$ the coefficients $a$ and $b$ are respectively $7.3 \cdot 10^{-8}$ and 3.25 .

Numerical modelling of the problem under consideration took into account only the processes of target heating, melting and evaporation. 2-D calculation was performed under a cylindrically symmetrical formulation on a non-uniform rectangular mesh with a $60 \times 60$ structure. The draft force of the target irradiated was calculated as

$$
F=\int_{S} P_{s} d S
$$


and the resultant momentum is given by the expression

$$
J=\int_{\tau} F d \tau .
$$

Here $P_{s}$ is the pressure upon the target surface area $d S$ (here one should take into account the target surface orientation), $\tau$ is the laser radiation pulse duration.

The calculations were accomplished for the laser pulse energies $E / S=$ 400,600 , and $1200 \mathrm{~J} / \mathrm{cm}^{2}$. The vapor recoil pressure values obtained are indicated with markers in Fig.11. The direct line in the figure corresponds to experimental dependence (26). In Figs.12-15, presented are the temperature profiles over the sample surface at different moments of time as well as the pressure profiles at different densities of incident laser radiation energ $\ddot{y}$. In addition, Fig.16 presents the shape of an erosional crater on the aluminum target at the laser pulse end. Unfortunately, in [12] neither the size of the erosional crater nor the temporal dependence of the pressure impulse is given, which would be rather helpful when comparing the experimental estimates to theoretical ones.

As a whole, it is worth noting a satisfactory agreement of the calculated and experimental dependences of vapor recoil impulse on incident radiation energy. Thus, the mathematical model of ablation (as well as its numerical realization) shows promise of describing adequately the actual physical processes taking place when moderate-intensity laser radiation interacts with matter. 


\section{Conclusion}

The report above presents a theoretical analysis of the regimes used for laser processing of materials under conditions of shallow heat and mechanical attack. Considered are the possible conditions of heat and mass transfer with taking into account the vapor pressure, material melting-through dynamics, and the surface tension versus temperature dependence. Presented are the dependences of ablation removal of material on parameters of laser beam interaction with matter. Discussed are the physical processes in the ambience, which prevent the direct use of the numerical code for calculation of material removal under high-intensity beam irradiation. The use of the numerical code for comparison with the experimental data on doping iron with molybdenum [13] and on dynamics of material removal due to läserinduced ablation of metals [12] revealed a satisfactory correlation between the numerical and experimental results.

A change to the range of higher laser beam power and energy densities invites further research into regimes of deep melting with formation of deep craters (deep penetration). In the report to follow we consider the cavern formation mechanisms and physical processes attendant to radiation propagation through narrow channels and waveguides. The mathematical model describing material removal from a deep cavern due to ablation and the corresponding numerical results will be presented as well. 


\section{References}

[1] John F.Ready. Industrial Application of lasers, Academic Press. New York, 1978.

[2] Vedenov A.A., Gladush G.G. Physical processes in Laser Machining of Materials, Moscow, Energoatomizdat, 1985.

[3] Arutyunyan R.V., Baranov V.Yu., Bol'shov L.A. et al. Laser beam material interaction, Moscow, Nauka, 1989.

[4] Levich V.G. Physical-Chemical Hydrodynamics, Moscow, Fizmatgiz, 1959.

[5] Gladush G.G., Krasitskaya L.S., Levchenko E.V., Chernyakov A.L. Kvantovaya Electronika, 1982, v.9, p.660.

[6] Anisimov S.I., Imas Ya.A., Romanov S.G., Khodyko Yu.V. High power laser beam interaction with metals, Moscow, Nauka, 1970.

[7] Anisimov S.I., Tribel'skii M.I., Enel'baum Ya.G. JETP, 1980, v.78.

[8] Tribel'skii M.I. Kvantovaya Electronika, 1978, v.63, p.586.

[9] Raizer Yu.P. Physics of gas discharge, Moscow, Nauka, 1987.

[10] Gill D.H., Dougal A.A. Phys.Rev.Lett., 1965, v.15, n.22, p.845.

[11] Barchukov A.I., Bunkin F.V., Konov V.I. et al. JETP, 1974, v.66, n.3, p.965.

[12] Kuznetsov L.I. Recoil momentum acting on a solid during intensive laserinduced evaporation: Kvantovaya Electronika, 1993, v.20, n.12, p.1191.

[13] Uglov A.A., Smurov I.Yu., Ignat'ev M.B. et al. Mass transfer under temperature gradient at laser doping from pre-deposited coating. Teplofizika Visokih Temperatur, 1990, v.28, n.3, p.530. 


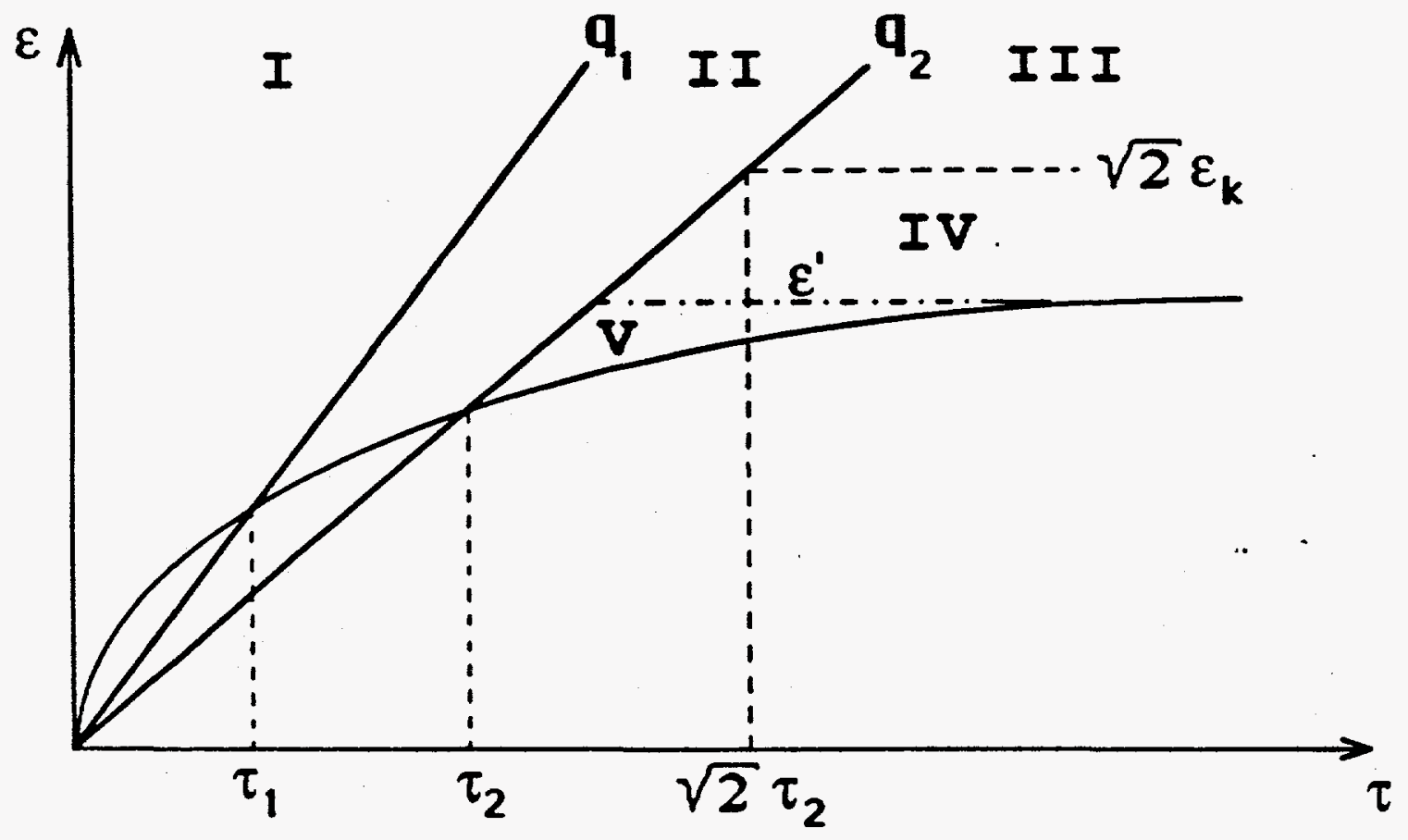

Fig.l Theoretical classiticalion of ine.lt removal. a absorbed energy density of laser bean during pube, $\tau$ - pulse duration (clarification see in text). 


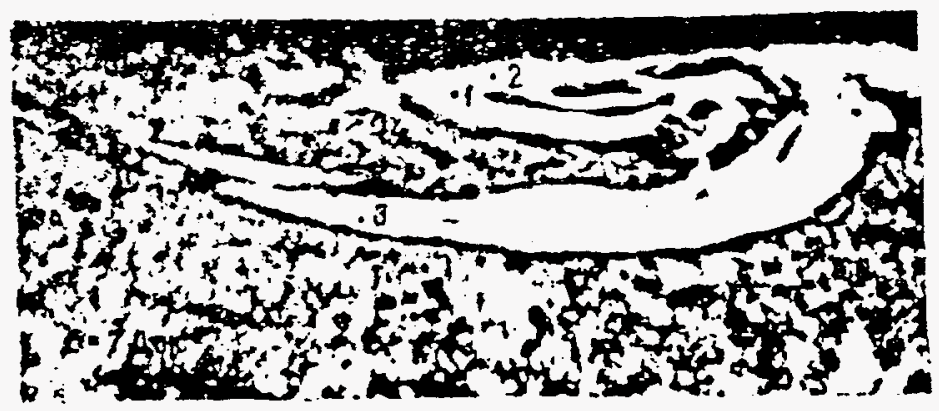

a

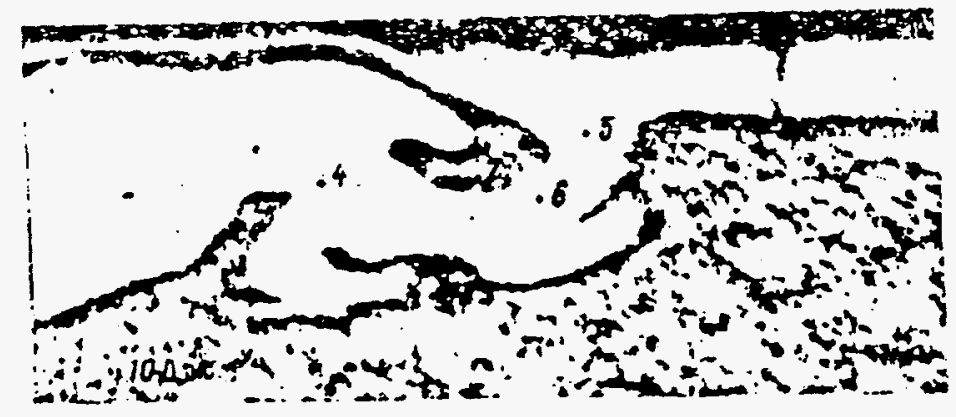

b

Fig. 2 Phoshegraples of the doped zune $\{13\}$ of two-layer system "iron molybdenum" at laner cuersit $E=7 J(a)$ and $E=10 J$ (b). Pre-cleposited nublybdenum coating $30 \mu \mathrm{m}$ thick, focal spot radius

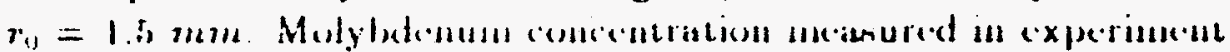
al puint $125 \%, 2$ 12\%, 3 $44 \%, 4 \quad 64 \%, 5-28 \%, 6-32 \%, 7-6 \%$. 


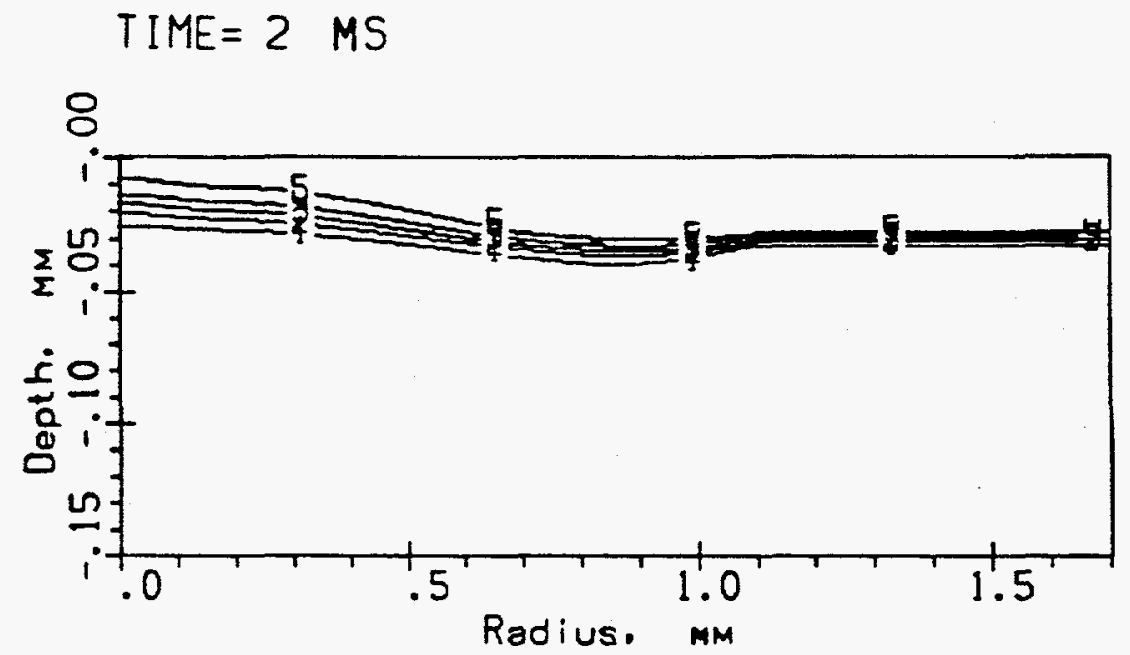

$C 1=0.1$

$C 2=0.3$

$C 3=0.5$

$C 4=0.7$

C $5=0.9$

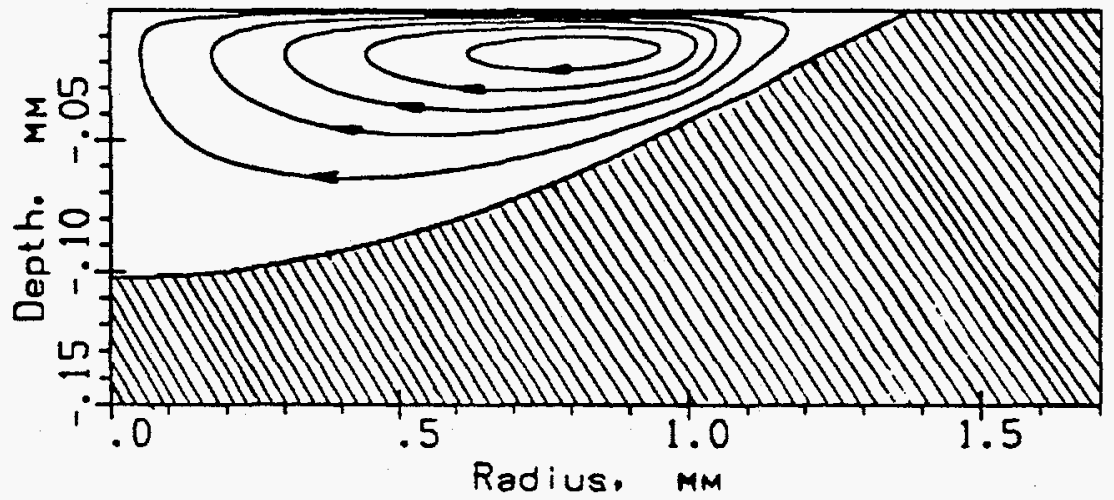

Fig. 3 Calculated melt bath shape, isolines of melt stream function and molybdenum concentration levels for moment $t=2 \mathrm{ma}$ at a laser radiation energy $F=10 \mathrm{~J}$. 

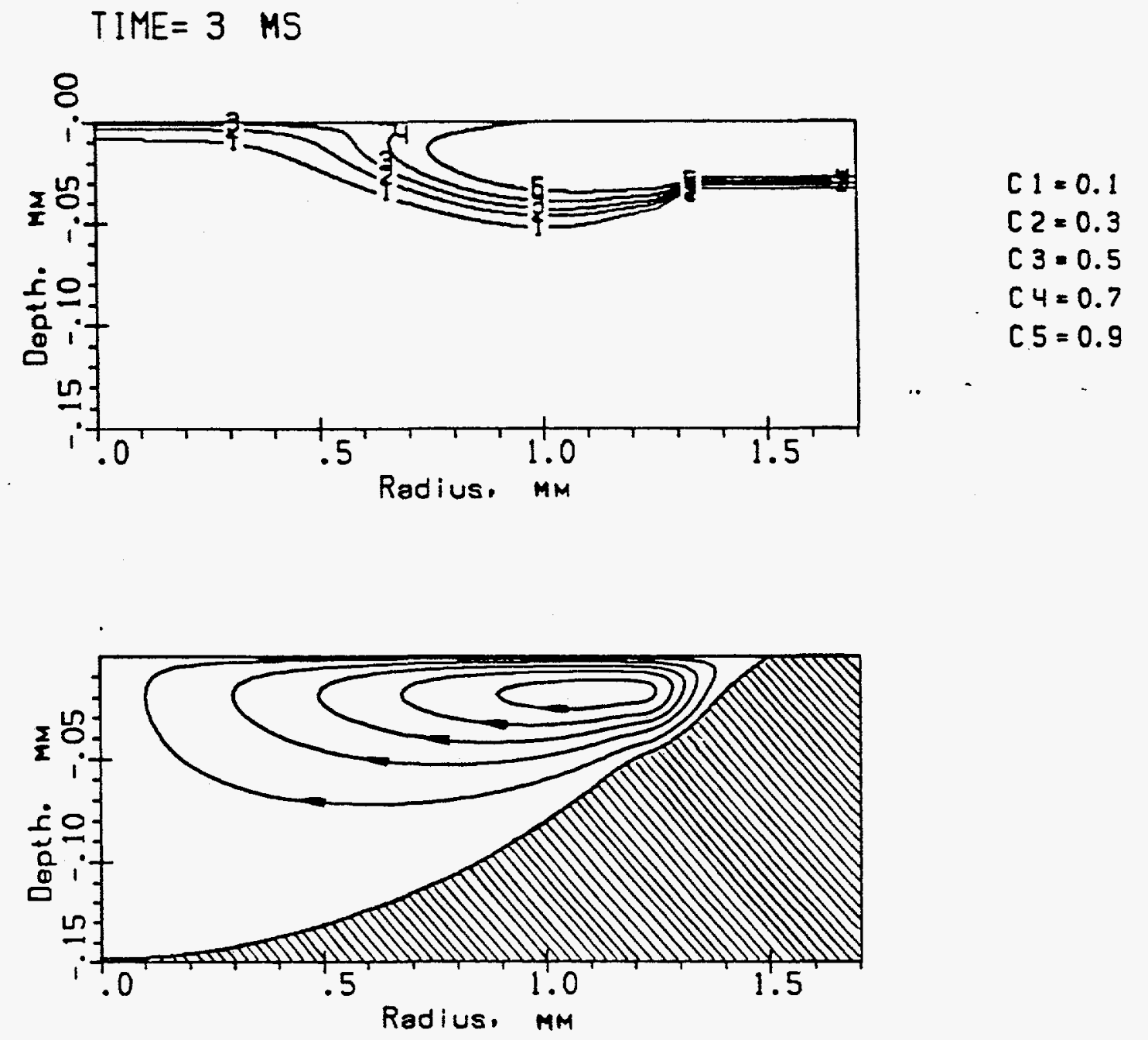

Fig.4 The rame care in Fig. 3 for moment $t=3 \mathrm{~ms}$. 


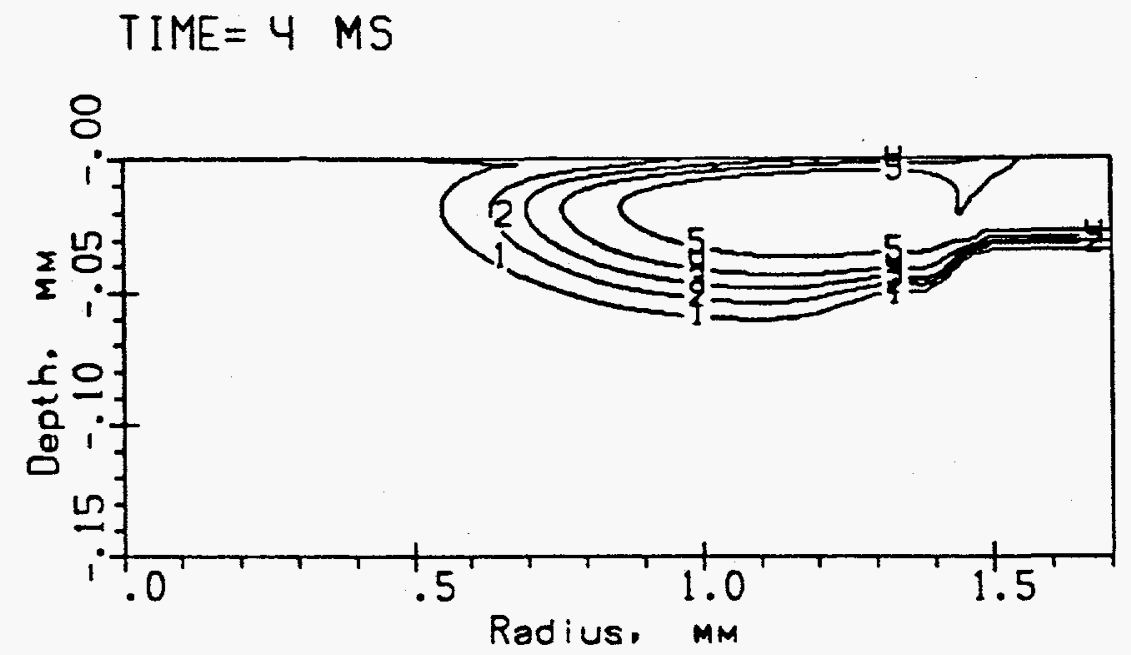

$C 1=0.1$

$C 2=0.3$

$C 3=0.5$

$C 4=0.7$

$C 5=0.9$

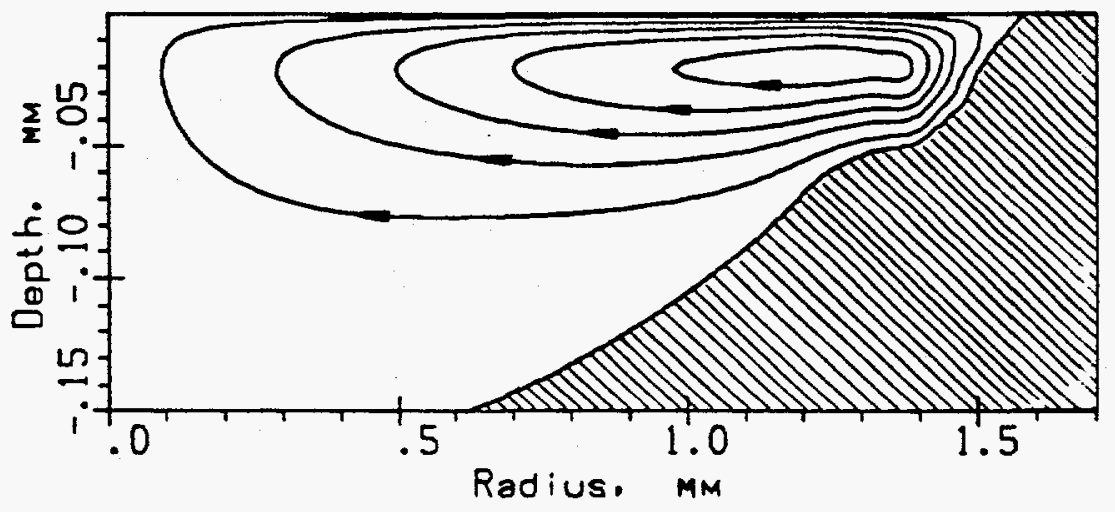

Fig.5 The same case as in Fig.3 for monsent $t=4$ ms. 

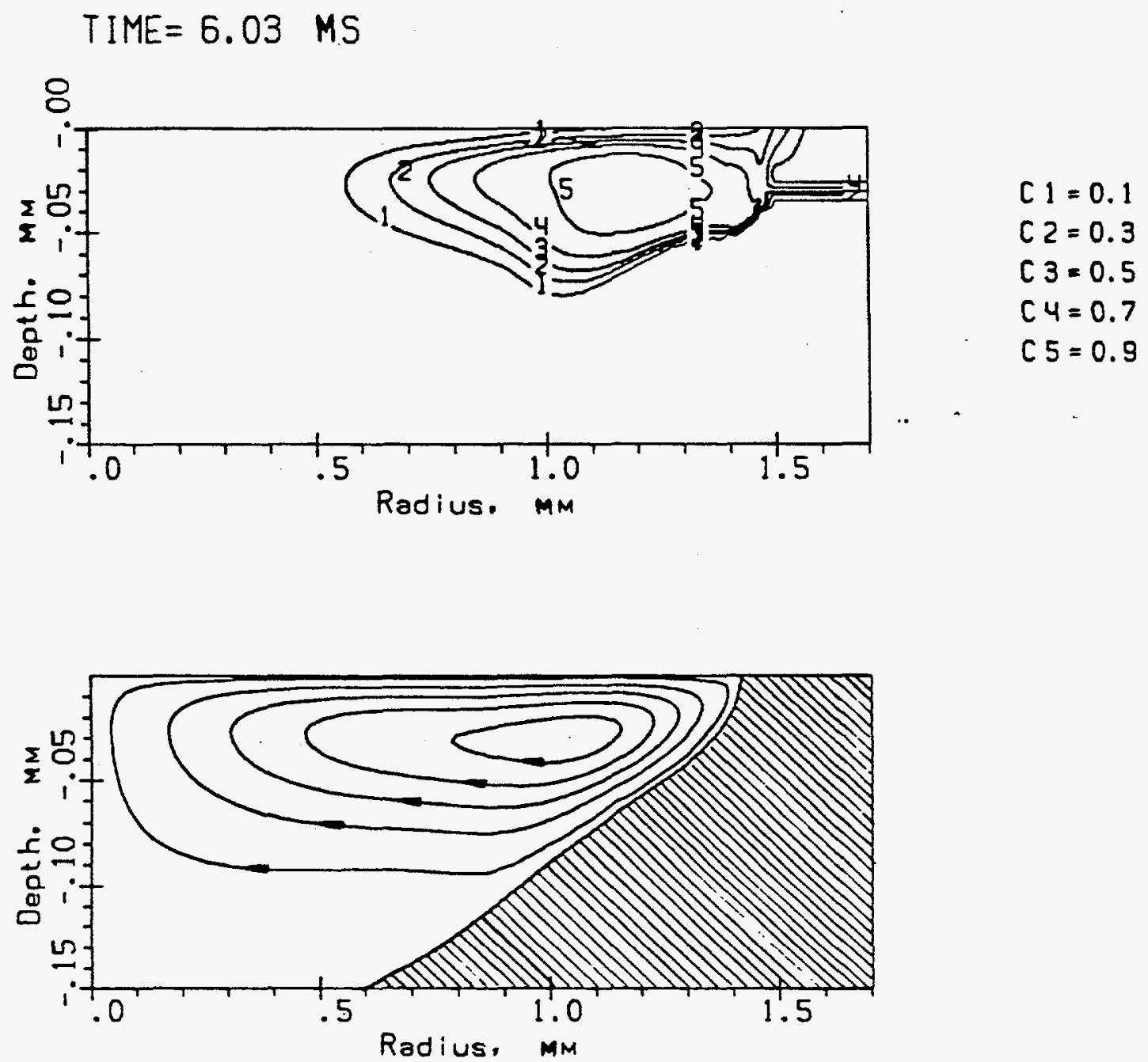

Fig.6 The same care as in Fig.3 for moment $t=6 \mathrm{~ms}$. 
TIME $=8.03 \mathrm{MS}$

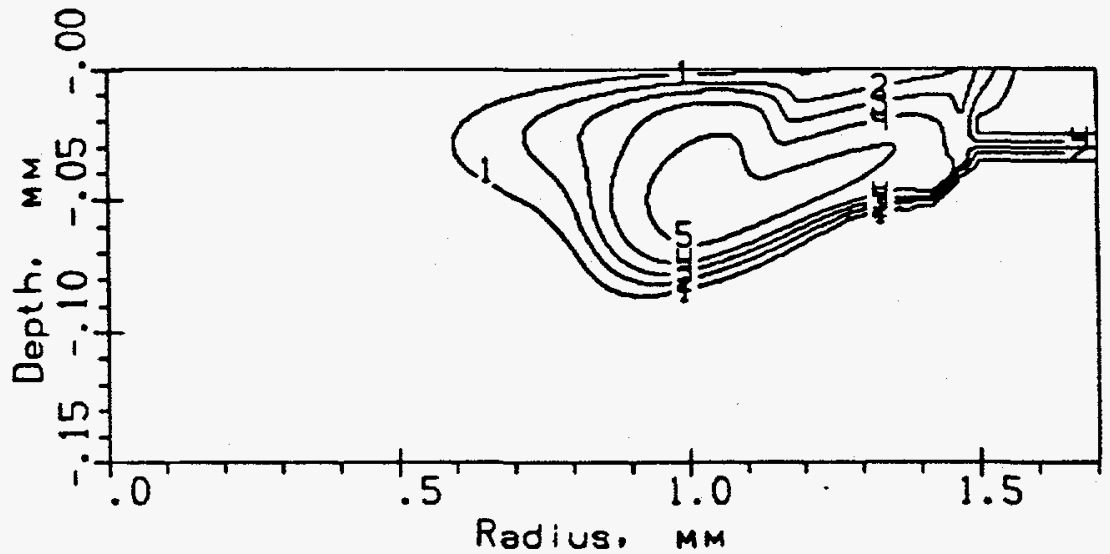

$C 1=0.1$

$C 2=0.3$

C $3=0.5$

$C 4=0.7$

C $5=0.9$

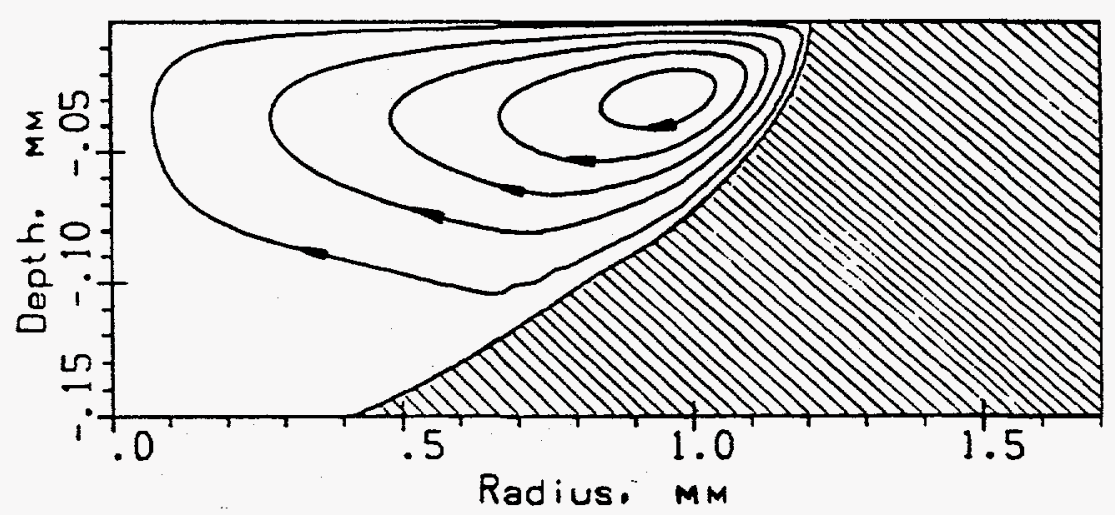

Fig.7 The same case as in Fig. 3 for moment $t=8 \mathrm{~ms}$. 


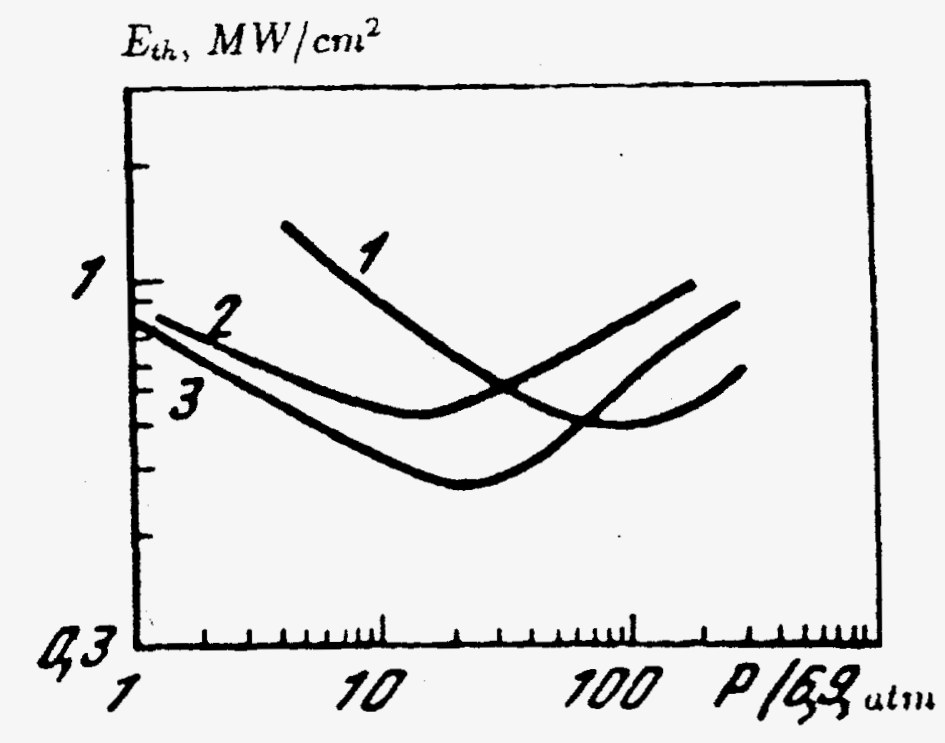

Fig.8 Dependence of breakdown threshold intensily of helium - (1), nitrogen $\cdots(2)$ and argon - (3) on pressure.

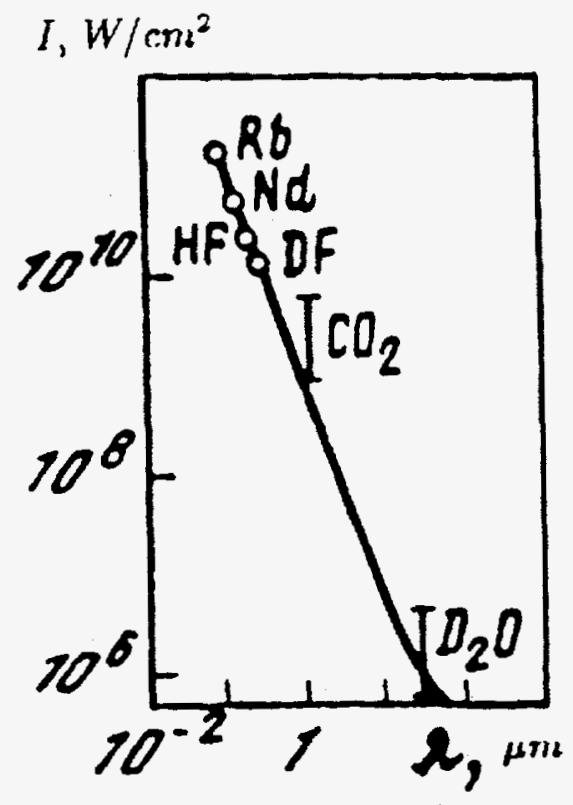

Fig.9 D.pendence of breakdown threshold intensity at atmospheric pressure air on radiation wavelength. 


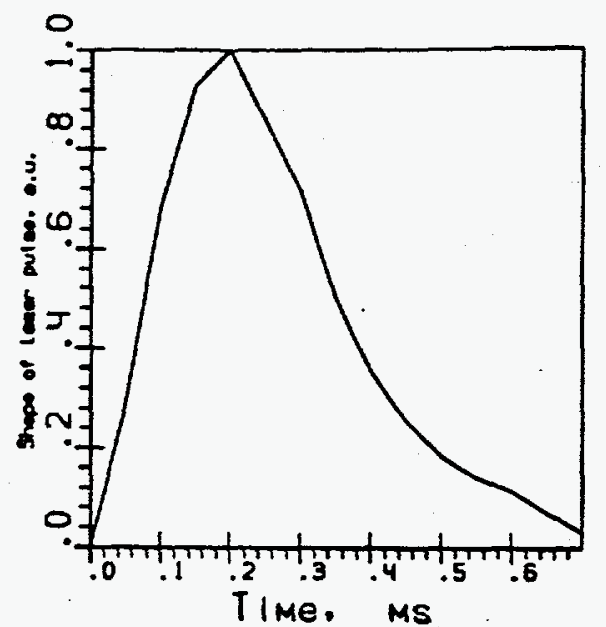

Fig. 10 'The laser pulse shape used in calculation.

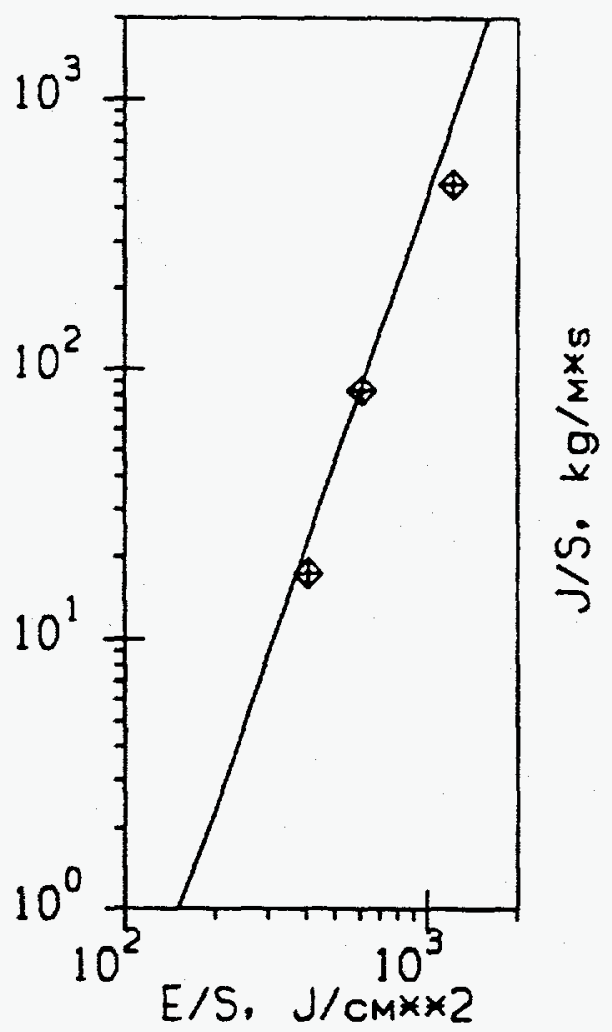

Fig.11 Dependence of vapor recoil pressure $J / S$ for aluminum target on laser energy $E / S$. The direct line - experinent [12], markers present calculation. 


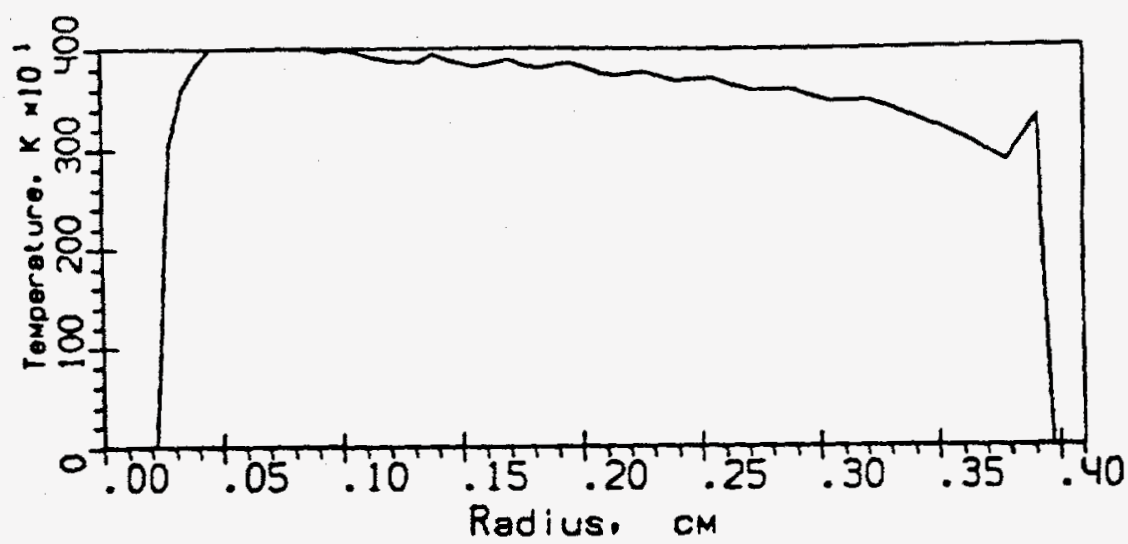

TIME $=0.30 \quad M S$

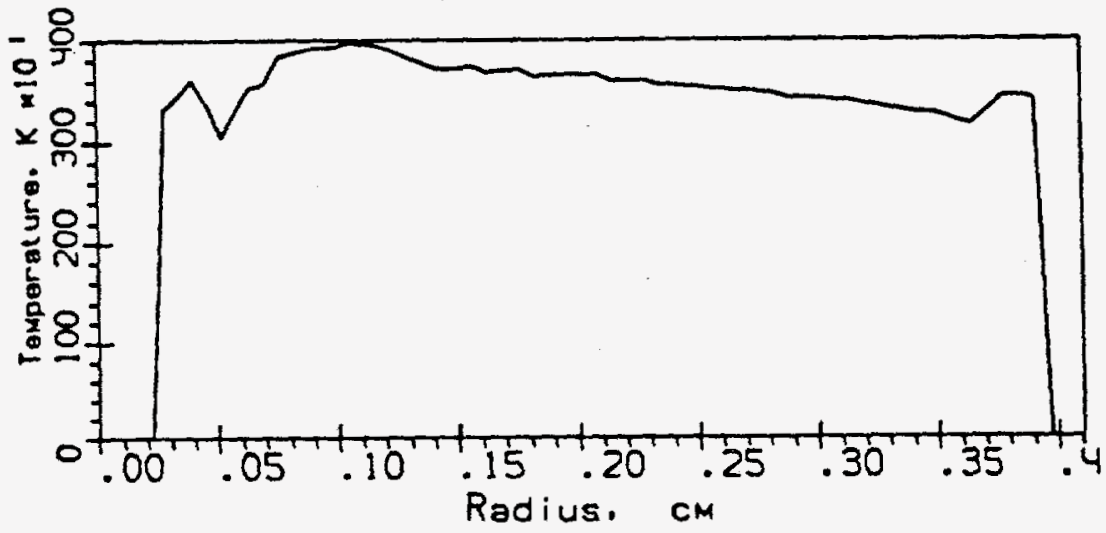

TIME $=0.40 \quad M 5$

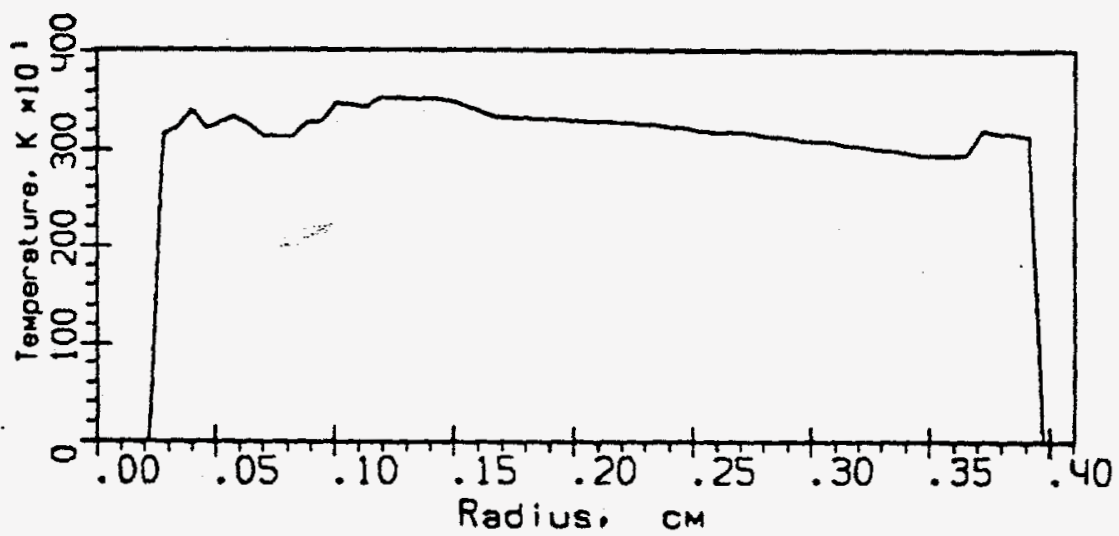

Fig. 12 The temperature profiles over the sample surface at different. moments of time at deneity of laser energy $k / S=1200 \mathrm{~J} / \mathrm{cm}^{2}$. 


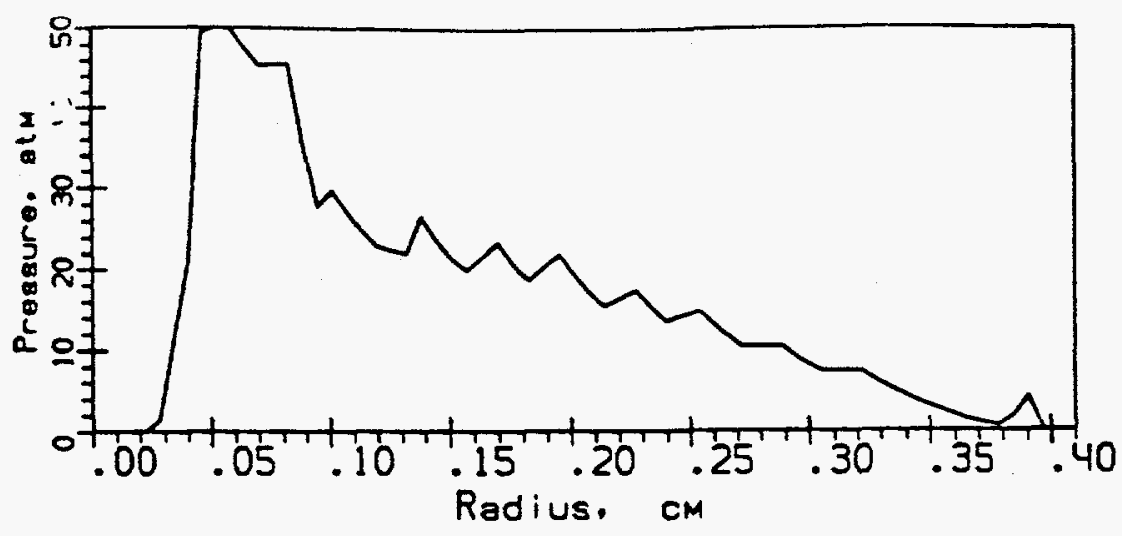

TIME $=0.30 \mathrm{MS}$

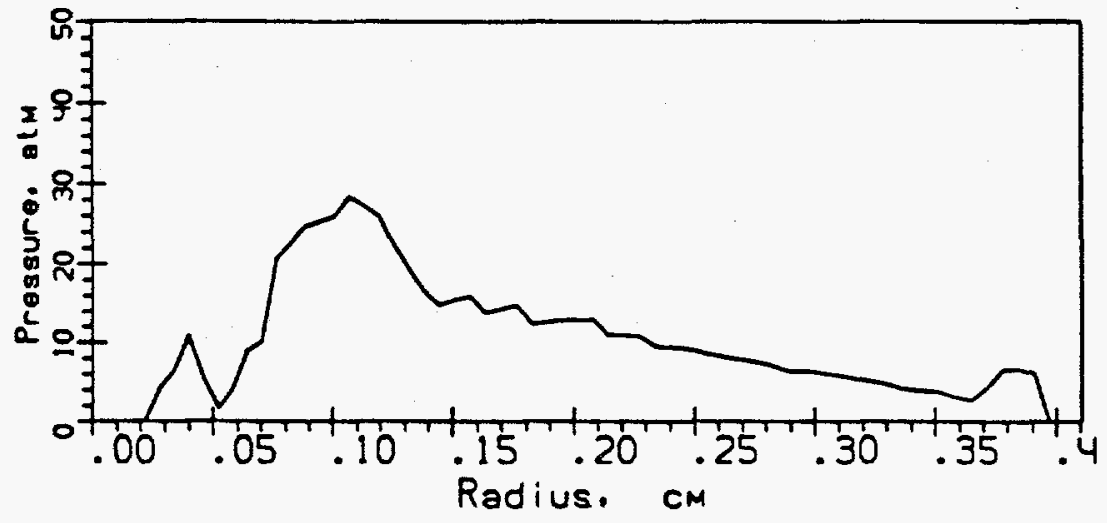

TIME $=0.40 \quad M S$

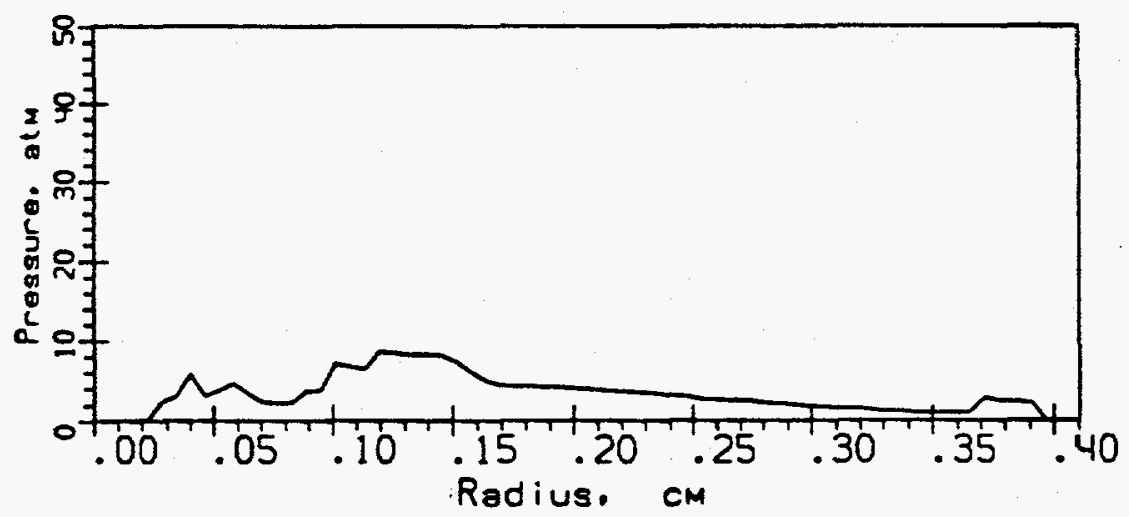

Fig.13 The pressure profiles over the sample surface at different moments of time at density of laser energy $E / S=1200 \mathrm{~J} / \mathrm{cm}^{2}$. 
TIME $=0.20 \mathrm{MS}$

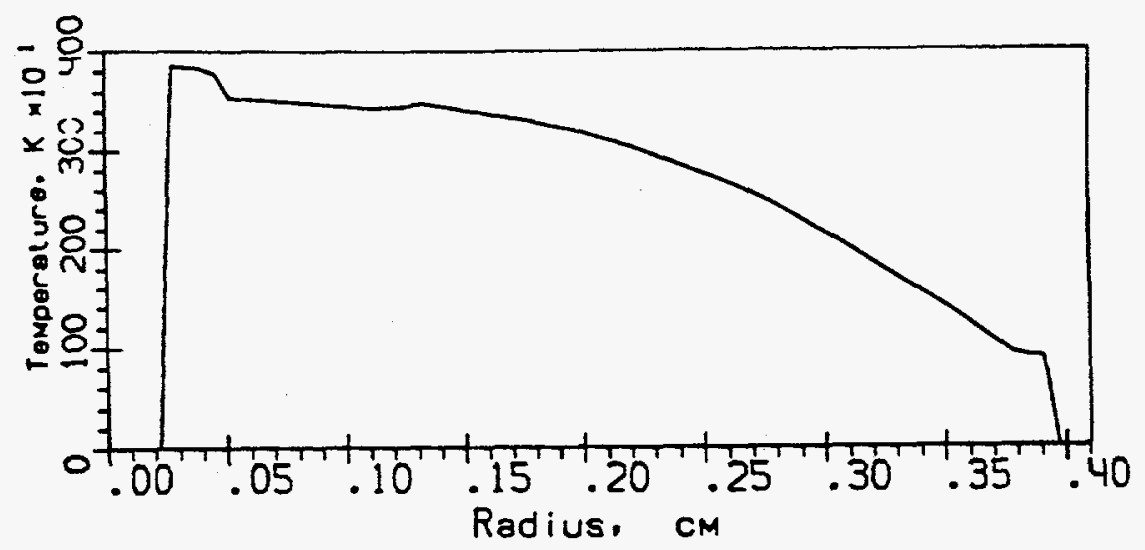

TIME $=0.30 \mathrm{MS}$

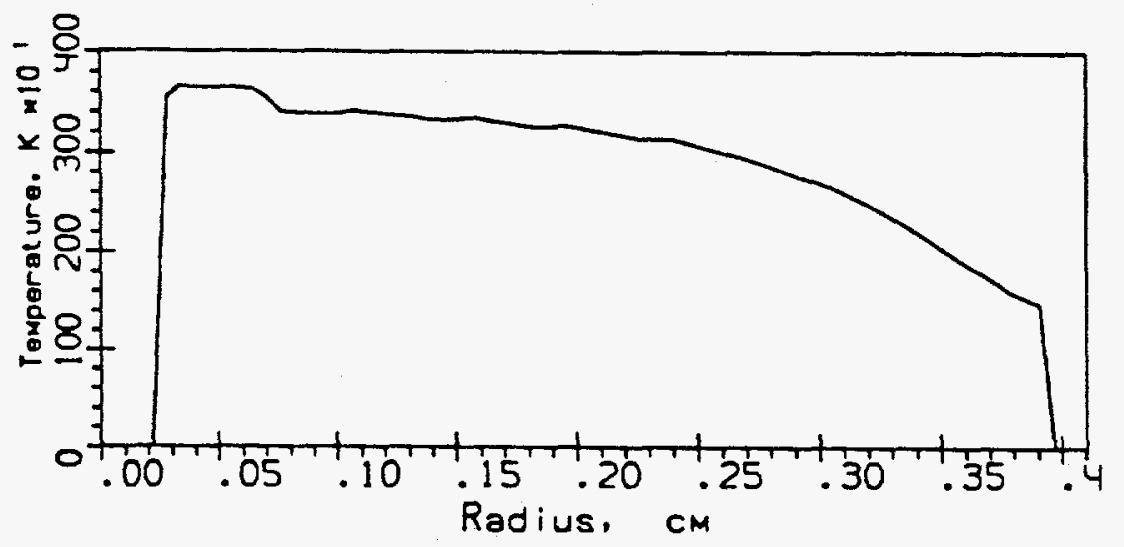

TIME $=0.40 \mathrm{MS}$

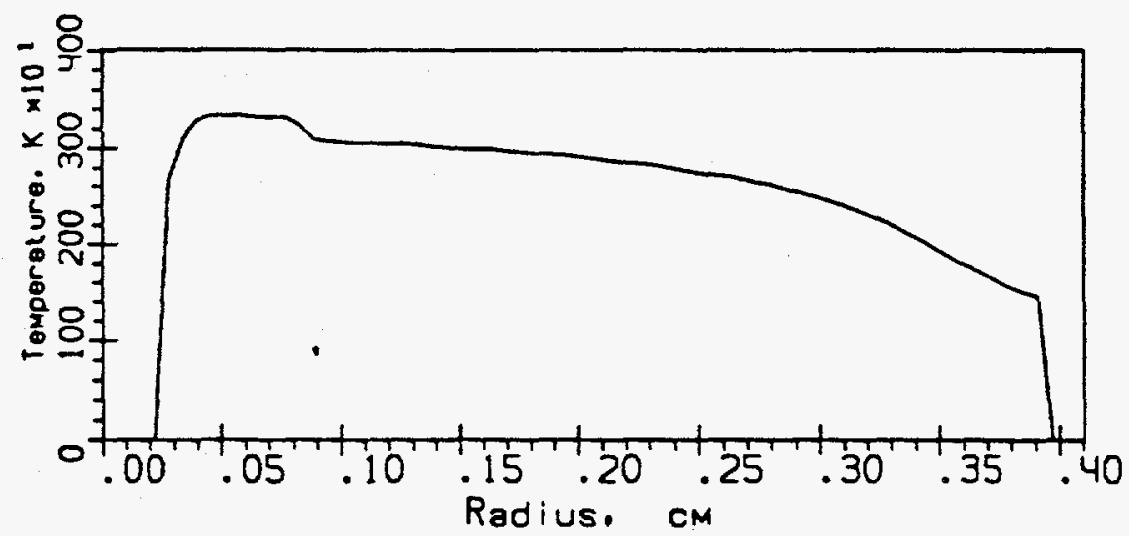

Fig.14 The temperature profiles over the sample surface at differcnt moments of time at density of laser energy $E / S=600 \mathrm{~J} / \mathrm{cm}^{2}$. 


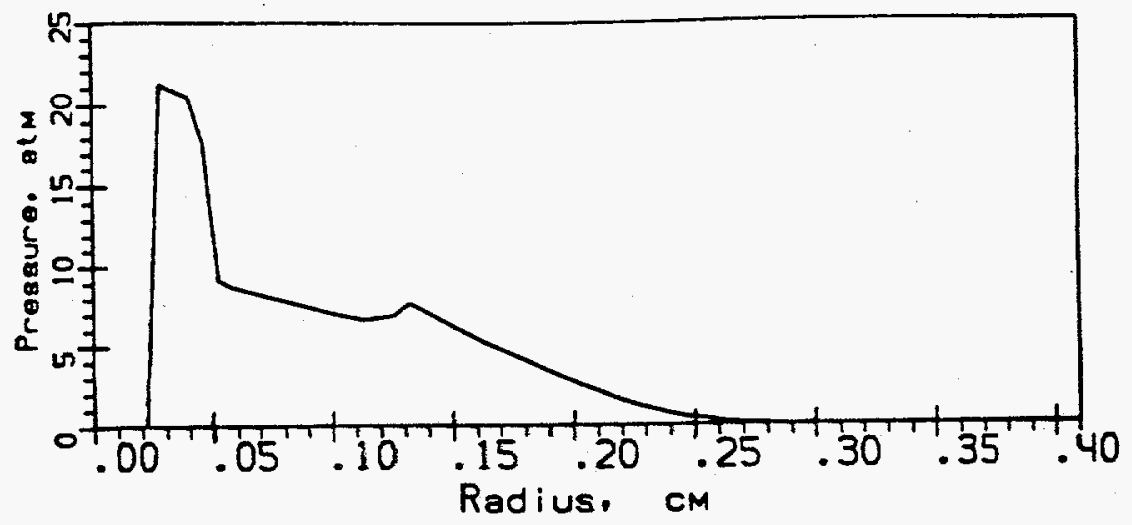

TIME $=0.30 \quad M S$

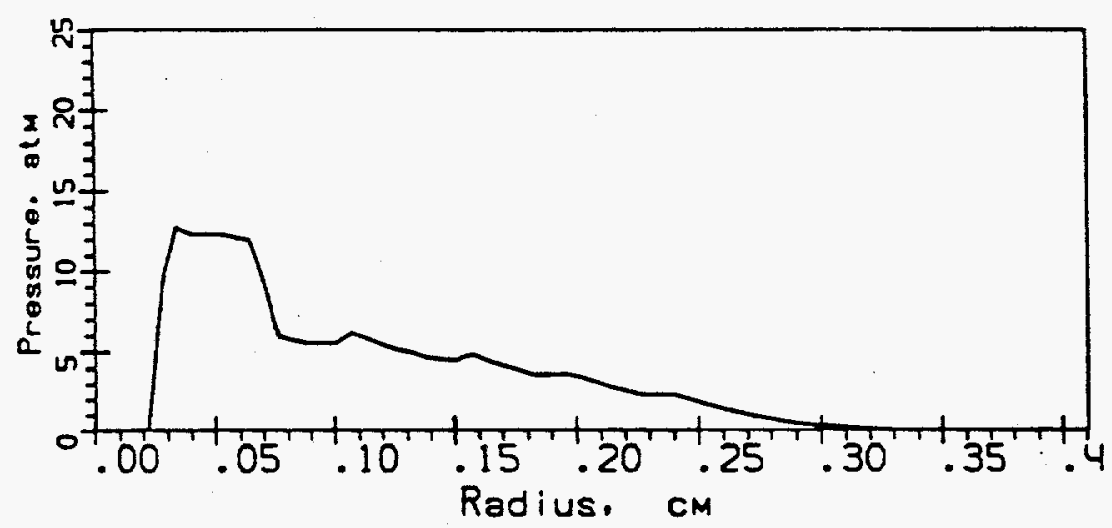

TIME $=0.40 \mathrm{MS}$

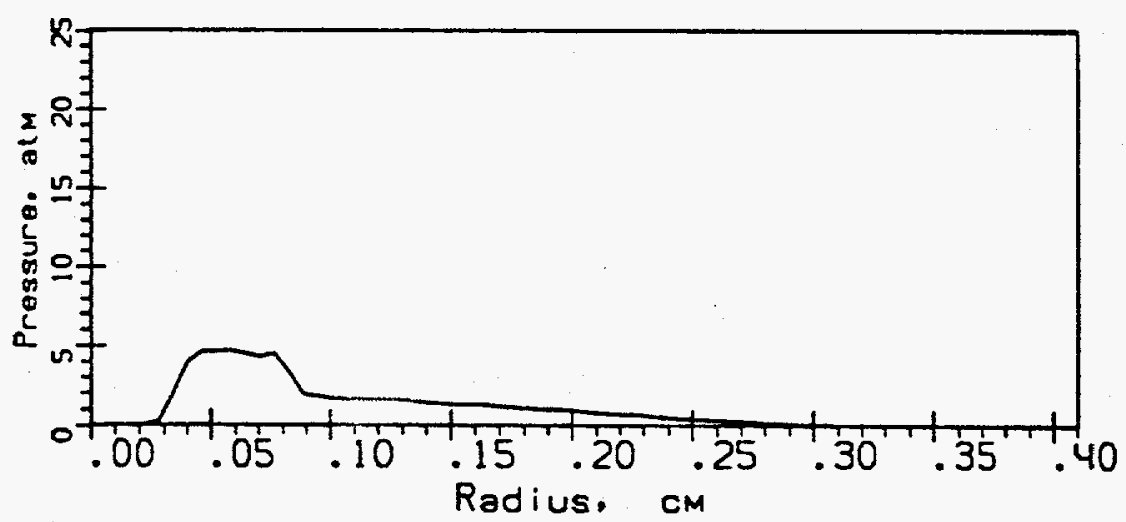

Fig.15 The pressure profiles over the sample surface al different. moments of time at density of laser energy $E / S=600 \mathrm{~J} / \mathrm{cm}^{2}$. 
$E / S=400 \Pi$ I $/ \mathrm{CM}^{2}$

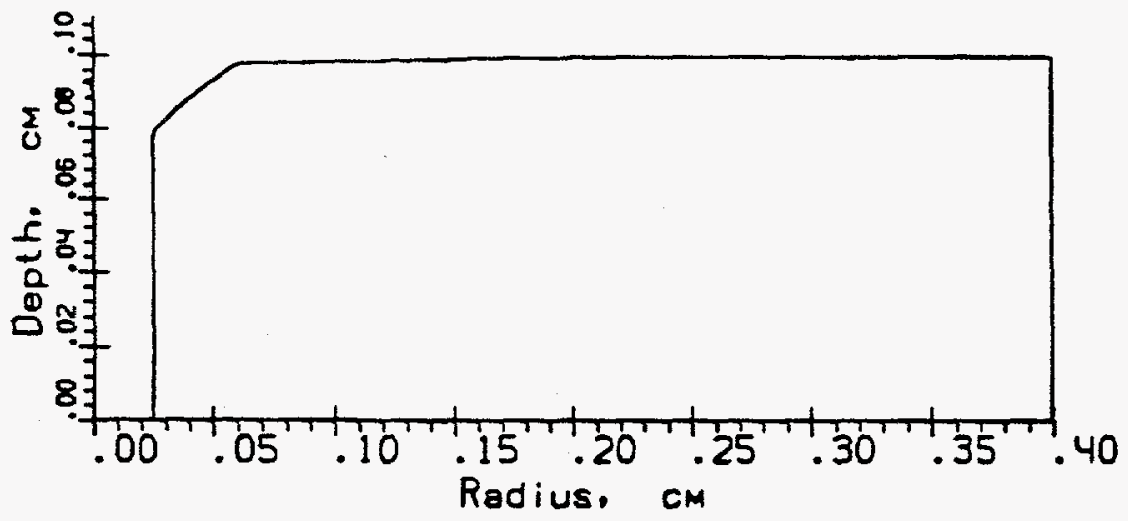

$E / S=600 \mathrm{I} \times / \mathrm{CM}^{2}$

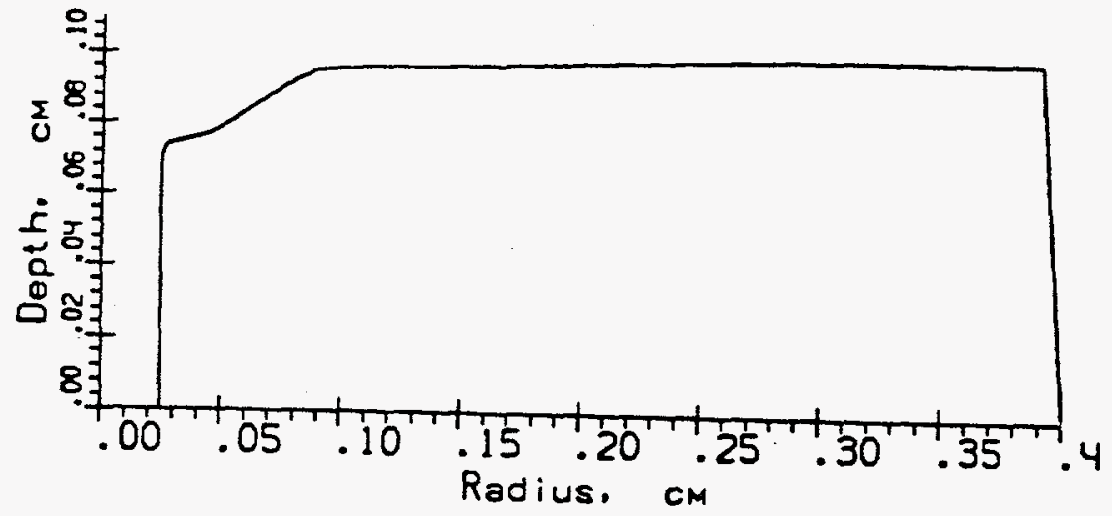

\section{$E / S=1200 \mathrm{I} \times / \mathrm{CM}^{2}$}

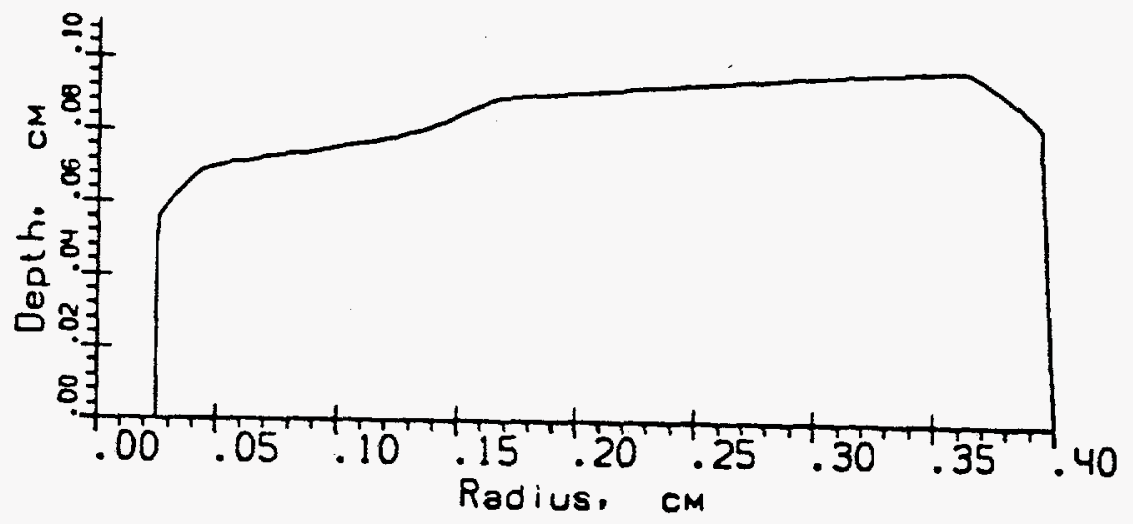

Fig. 16 'The shapes of an erosional crater on the aluminum target at the laser pulse end for different densities of laser energy. 